\title{
DFT and TD-DFT study on quadratic NLO response and optoelectronic activity in novel Y-Shaped imidazole-based push-pull chromophores
}

Lamia Kara Zaitri

Universite Abou Bekr Belkaid Tlemcen

Sidi Mohamed Mekelleche ( $\nabla$ sidi_mekelleche@yahoo.fr)

Universite Abou Bekr Belkaid Tlemcen https://orcid.org/0000-0002-5806-3333

\section{Research Article}

Keywords: Imidazole derivatives, Nonlinear optics (NLO), Charge transport properties, Luminescent materials, Organic light-emitting diodes (OLEDs), DFT and TD-DFT calculations

Posted Date: February 16th, 2021

DOI: https://doi.org/10.21203/rs.3.rs-197164/v1

License: (c) (i) This work is licensed under a Creative Commons Attribution 4.0 International License.

Read Full License 


\title{
DFT and TD-DFT study on quadratic NLO response and optoelectronic activity in novel Y-Shaped imidazole-based push-pull chromophores
}

\author{
Lamia Kara Zaitri and Sidi Mohamed Mekelleche* \\ Laboratory of Applied Thermodynamics and Molecular Modeling, Department of Chemistry, Faculty of \\ Science, University of Tlemcen, PB 119, Tlemcen, 13000, Algeria \\ *Email of the corresponding author: sm_mekelleche@mail.univ-tlemcen.dz
}

\begin{abstract}
A theoretical analysis of a series of imidazole-based Y-shaped chromophores, D1-D8, is performed in order to investigate their non(linear) optical, fluorescence, and charge-transport properties. The calculations have been carried out employing DFT and TD-DFT methods at CAM-B3LYP and M06-2X levels of theory. FMO analysis reveals that in ground state, highest occupied molecular orbital is localized on the 4,5-dimethylanilino donor moiety and imidazole core while lowest unoccupied molecular orbital spreads on $\pi$-linker and nitro acceptor moieties. Vertical absorption and fluorescence transitions are characterized as intramolecular charge transfer and maximum absorption and fluorescence wavelengths show that by changing the $\pi$-bridge to the imidazole $\mathrm{C} 2$, we can tune fluorescence color from cyan to orange. Calculated (hyper)polarizabilities show that elongation of $\pi$ linker by polarizable subunits, such as double bonds or heteroaromatic rings, increases significantly the nonlinear response and shifts the charge-transfer band bathochromically. Calculated reorganization energies indicate that the studied compounds are hole-transporting materials rather than electron-transporters. Interestingly, D7 and D8, with higher hyperpolarizabilities, are predicted to be potent candidates for NLOdevices while D5 and D8 molecules are expected to be promising candidates for luminescent materials and good hole-transport materials for organic light emitting diodes.
\end{abstract}

\section{Keywords}

Imidazole derivatives; Nonlinear optics (NLO); Charge transport properties; Luminescent materials; Organic light-emitting diodes (OLEDs); DFT and TD-DFT calculations 


\section{Introduction}

Due to their ease of synthesis and structural modification, organic dyes are widely studied [1]. They exhibit high chemical and good thermal robustness, large optical transparency wavelength range, high thresholds for laser damage and good solubility in common organic solvents [2, 3]. Furthermore, they have considerable potential as active components in the design of devices for telecommunications, optical data storage and optoelectronic, such as light-emitting diodes (OLEDs), and organic photovoltaic cells [4, 5]. Both the (non)linear optical response of the molecule and the efficiency of charge transport within organic layers play a crucial role in these applications, $[6,7]$.

Considerable attention has been focused over the last few decades and significant progress has been made in the development of new organic push-pull systems [8] exhibiting organic light emitting properties or large NLO susceptibilities [9-11]. A typical organic push-pull chromophore consists of strong electron donor D and strong electron acceptor A linked by a $\pi$-conjugated system [12]. This D- $\pi$-A arrangement ensures efficient intramolecular charge transfer (ICT) between the donor and acceptor moieties and generates a dipolar system with low-energy and intense charge transfer absorption. Chromophores containing two donor groups $\left(\mathrm{D}_{2}-\pi-\mathrm{A}\right)$ display improved intramolecular charge transfer compared to D- $\pi$-A molecules with one donor group [13].

Numerous experimental and theoretical studies carried out over the past few decades have shown that optimal molecular hyperpolarizabilities can be achieved by tuning the electronic nature and length of the $\pi$-conjugated linker $[14,15]$ and/or the strength of the attached donor/acceptor electron groups [16]. In particular, the relationship between structure and property indicates that the molecular hyperpolarizability depends on the $\pi$-system building blocks, such as (hetero)aromatic rings and double-bonded fragments [9]. For the construction of suitable $\pi$-bridge in charge transfer chromophores, imidazoles have proved to be highly efficient five-membered heteroaromatics [17-19].

Molecules based on imidazole typically have donor/acceptor groups connected by $\pi$-conjugated linker to form two main classes of Y-shaped charge transfer chromophores YCTCs [20]. The acceptor is appended to the imidazole $\mathrm{C} 2$ through an additional $\pi$-linker in the class denoted A- $\pi$-IM- $(\pi-\mathrm{D})_{2}$, and two peripheral donors are linked at the imidazole C4 and C5 positions. In reverse orientation, the class, denoted D- $\pi$-IM- $(\pi \text {-A })_{2}$, has one donor and two acceptors In particular, 4,5-dimethyl-anilinoimidazole chromophore derivatives have been widely used for the construction of promising charge-transfer chromophores as suitable and robust aromatic systems $[21,22]$. In addition, these materials exhibit respectable dipole moments, substantial nonlinearities, and retain significant polar order even at high temperature [23]. Furthermore, it was found that chromophores based 
on the imidazole core were extensively studied as photoluminescence materials and showed strong and tunable solid-state blue emissions, making them potentially applicable to OLEDs as active layers[24].

In the present work, quantum chemical methods based on density functional theory (DFT) [25] and time-dependent DFT (TD-DFT) [26] have been used in order to better understand and evaluate the effects of the electronic nature and length of $\pi$-conjugation on (non)linear optical and charge transport properties in a series of substituted triaryl imidazole derivatives D1-D3 (Scheme 1a) as well as planarized imidazole derivatives, phenanthro[9,10]imidazoles D4-D8 (Scheme 1b). Moylan et al. [23] found that the planarization of the 4,5donor rings by the formation of a polycyclic phenanthrene aromatic core significantly increases the measured quadratic molecular hyperpolarizability [21]. The chromophore D1 [27] is the 2,4,5-triarylimidazole substituted with an electron-donating group $\left(\mathrm{NMe}_{2}\right)$ at the para positions of the 4-aryl and 5-aryl rings and an electronwithdrawing group $\left(\mathrm{NO}_{2}\right)$ at the para position of the 2-aryl ring. Compared to D1, the positions of the donor/acceptor groups are inversed in D2. In D3 studied by Pokladko-Kowar et al. [28], the ethylenephenyl unit is added to the D1 $\pi$-linker. The 3,6-disubstituted2-phenylphenanthro[9,10]imidazole D4 is the planar analogue of $\mathrm{D} 1$. In the phenanthro[9,10]imidazole derivatives $\mathrm{D} 5-\mathrm{D} 8$, the $\mathrm{NO}_{2}$ group is linked to the imidazole core via various conjugated $\pi$-systems where double bonds as well (hetero)aromatic rings are introduced (see Scheme 1a-b).

Our aim in the present contribution is to investigate the electronic and optical properties in a series of YCTCs D1-D8 in order to design novel potent imidazole-based compounds with substantial molecular hyperpolarizabilities, strong luminescence with tunable colour as well as high charge transporting characteristics for NLO and OLED devices.

\section{$<$ Insert Scheme 1 here $>$}

\section{Computational details}

All gas phase equilibrium geometries of chromophores D1-D8 were fully optimized, without any symmetry constraints, using the Berny analytical gradient optimization method [29] and the standard polarized split-valence 6-31G(d,p) basis set [30] implemented in the Gaussian 09 package [31]. The true minima were checked by the absence of imaginary harmonic vibration frequencies in the Hessian Matrix. The ground states (S0) geometries were optimized using the long-range corrected CAM-B3LYP functional [32] which has been successfully used for $\pi$-conjugated systems [33]. The first excited singlet states (S1) structures were optimized using the TD-CAM-B3LYP method which has been proven efficient in the determination of the charge-transfer transition energies for organic conjugated chromophores [34]. The Coulomb-attenuating method CAM-B3LYP 
[32] and the Minnesota functional M06-2X [35] in conjunction with 6-311++G(d,p) basis set were used for investigating the gas phase second-order NLO behaviors of the studied chromophores. These two functionals are currently more preferred than the traditional functionals to predict the NLO properties of large $\pi$-conjugated systems with a significant CT effect [36-38]. In this work, we used the sum-over-states (SOS) method [39] and the two-state model (TSM) [40], based on electronic absorption characteristics, in order to relate the NLO responses of the studied compounds to their linear optical properties. The electronic absorption and fluorescence spectra in chloroform were predicted employing single point calculations performed with the TD-CAM-B3LYP and TD-M06-2X methods in conjunction with the 6-31G++(d,p) basis set. The S0 and S1 optimized states were used for the calculation of absorption and fluorescence characteristics, respectively. These two functionals are found to be appropriate for calculating the absorption and fluorescence spectra of $\pi$-conjugated organic chromophores [41, 42]. For instance, the calculated absorption $\lambda_{\text {abs }}$ and emission $\lambda_{\text {flu }}$ wavelengths of D3 agree well with the available experimental data [28] (see Tables 4-5). Solvent dielectric effects were considered using the popular implicit conductor-like polarizable continuum model (C-PCM) [43] which has become an increasingly useful tool for predicting the electronic structures of ground and excited states of molecules in solution [44]. The internal reorganization energies, which is one of the important parameters for determining the charge transporting properties, have been calculated in the gas phase at the CAM-B3LYP/6-31G(d,p) [45] level for the studied YCTCs D1-D8.

\section{Results and discussion}

\section{Frontier molecular orbitals}

It will be helpful to analyse the frontier molecular orbitals (FMOs) of the molecules since the relative ordering of the occupied and virtual orbitals gives a qualitative indication of the excitation properties and of the ability of electron or hole transportation [46]. A molecule with a smaller frontier orbital gap is a soft molecule and consequently easily polarizable.

Figure 1 displays the contour plots of the highest occupied molecular orbitals (HOMOs) and the lowest unoccupied molecular orbitals (LUMOs). It turns out that the electronic cloud distribution of the HOMOs of D1D8 are quite similar and they are predominantly located on the strong electron-donor units as $\pi$-bonding orbitals. Note that, the central imidazole moiety behaves as an electron donor in all systems. However, the electronic cloud distribution of the LUMOs of D1-D8 are extended over the $\pi$-conjugated linker and $\mathrm{NO}_{2}$ moieties as $\pi$ antibonding orbitals. It means that the charge transfer in D1-D8 occurs from the imidazole-to- $\mathrm{NO}_{2}$ which leads 
to an efficient intramolecular charge transference and charge separation. We note that the extended $\pi$ conjugation lengths in D3-D8 cause a significant difference in LUMO distribution compared with that in the reference system D1.

$<$ Insert Figure 1 here $>$

The energies of the HOMOs, LUMOs, the HOMO-LUMO energy gaps, $\Delta \mathrm{E}_{\mathrm{H}-\mathrm{L}}$, for the ground state structures D1-D8 are calculated at the CAM-B3LYP/6-31G(d,p) level of theory and given in Table 1 . The values of the HOMO and LUMO energies, in eV, vary in the ranges [-6.91, -5.94] and [-2.48, -1.52] respectively. We found that, except for D2, the HOMO energies of YCTCs are slightly changed (-6,17 eV for D1, -6,04 eV for D3, 6,11 eV for D4, -5,96 eV for D5, -6,01 eV for D6, -5,94 eV for D7, and -6,07 eV for D8); whereas the LUMO energies of D3-D8 YCTCs are all lower than that of D1. The impact of the additional units in $\pi$-conjugated spacer is reflected in the stabilization of the LUMOs, which is responsible for the enhancement of ICT in these systems. Table 1 shows that the extended $\pi$-conjugation length in D7 and D8 can stabilize the LUMO energy by $0,53 \mathrm{eV}$ and $0,87 \mathrm{eV}$, respectively, resulting in the narrow HOMO-LUMO energy gap compared with the case for D4. This can be explained by the presence of the ending thiazole moiety which acts as an auxiliary acceptor group.

$<$ Insert Table 1 here $>$

\section{NBO analysis}

Molecular orbital wave functions are transformed into one- and two-center representations by the natural bond orbital NBO method [47]. It gives insight into the interactions between different parts of the molecule. The NBO method provides information about interactions of orbital spaces, both filled and virtual, that could improve the study of intra- and intermolecular interactions. It is also possible to analyze hyperconjugative interactions due to electron transfer from filled bonding orbitals (donor) to empty anti-bonding orbitals (acceptor) [48]. The second-order Fock matrix was performed to evaluate the donor-acceptor interactions on the basis of NBO. The interactions contribute to a loss of occupancy from the idealized Lewis structure's localized NBO into an empty non-Lewis orbital. The stabilization energy $\mathrm{E}^{(2)}$ associated with the delocalization $\mathrm{i} \rightarrow \mathrm{j}$ is calculated, for each donor (i) and acceptor ( $\mathrm{j}$ ), as : 


$$
E^{(2)}=\varepsilon_{i j}=q_{i} \frac{F_{i j}^{2}}{\varepsilon_{j}-\varepsilon_{i}}=q_{i} \frac{\langle i|F| j\rangle^{2}}{\varepsilon_{j}-\varepsilon_{i}}
$$

where $q_{i}$ is the orbital occupancy of the donor; $\varepsilon_{i}, \varepsilon_{j}$ are the diagonal elements and $F_{i j}$ is the off-diagonal NBO Fock matrix element. In NBO analysis, strong interactions between electron-donors and electron-acceptors are shown by large $E^{(2)}$ values, and the extent of conjugation of the whole system is greater. The calculated stabilization energies $E^{(2)}$ for chromophores D1-D8 are presented in Table 2. It is important to note that the stabilization energies, $\mathrm{E}^{(2)}$, are proportional to the inverse of HOMO-LUMO gaps, $\Delta \mathrm{E}_{\mathrm{H}-\mathrm{L}}$ (see Figure 2). In Table 2 are given the natural charges of the different segments of chromophores D1-D8. The positive values for donor moieties well validate their electron-donating ability, while the negative values for the acceptor group $\mathrm{NO}_{2}$ reveal its electron-withdrawing ability. Moreover, the positive values for $\pi$-linkers indicate that they play the role of bridges which facilitate the charge transfer from donor to acceptor segments. These results point out that electrons move effectively from donor to acceptor through $\pi$-conjugated spacers and a charge separation state is formed.

\footnotetext{
$<$ Insert Table 2 here $>$

$<$ Insert Figure 2 here $>$
}

\section{Linear and nonlinear optical properties}

In designing materials commonly used for signal processing, communication technology, optical memory devices, and optical switches, the understanding of (non)linear optical properties is essential [49]. Electromagnetic radiation interaction with a molecule polarizes the charge distribution and alters the propagated field. The intensity of the optical response depends on the whole material's electronic properties. (Hyper)polarizability defines a molecule's (non)linear response. Therefore, in order to determine the optical ability of molecular systems, these quantities are the properties that should be computed.

The microscopic polarizability $(\mathrm{P})$ induced in an isolated molecule by an electromagnetic wave incident under the applied electric field (E) can be expressed by the following equation:

$\mathrm{P}=\alpha \mathrm{E}+\beta \mathrm{EE}+\gamma \mathrm{EE} \mathrm{E}+\ldots$

where $\mathrm{P}$ and $\mathrm{E}$ are related to the polarizability and first and second-order hyperpolarizabilities, respectively, of the tensor quantities $\alpha, \beta$ and $\gamma$. 
The polarizability is the ability of a molecule to be polarized and the average polarizability $\langle\alpha\rangle$ is determined by considering only the diagonal elements:

$\langle\alpha\rangle=\left(\alpha_{\mathrm{xx}}+\alpha_{\mathrm{yy}}+\alpha_{\mathrm{zz}}\right) / 3$

The first-order hyperpolarizability, $\beta$, is associated with the ICT through the $\pi$-conjugated system. $\beta$ is a third rank tensor that can be described by a $3 \times 3 \times 3$ matrix. Using the finite field theory approach, the value of $\beta$ is computed by using the $x, y$ and $z$ components of first order hyperpolarizability $\beta$. The magnitude of $\beta$ is calculated using following formula:

$\beta_{\text {tot }}=\left(\beta_{\mathrm{x}}^{2}+\beta_{\mathrm{y}}^{2}+\beta_{\mathrm{z}}^{2}\right)^{1 / 2}$

where $\beta_{\mathrm{i}}$ is defined as follows:

$\beta_{\mathrm{i}}=\frac{1}{3} \sum_{\mathrm{j}}\left(\beta_{\mathrm{ijj}}+\beta_{\mathrm{jji}}+\beta_{\mathrm{jij}}\right) \quad \mathrm{i}, \mathrm{j} \in\{\mathrm{x}, \mathrm{y}, \mathrm{z}\}$

Moylan and co-workers [21,23] contributed significantly to the field of imidazole-derived CT chromophores for NLO. They showed that the chromophore D1 (Scheme 1) and its derivatives are characterized by substantial optical nonlinearities, high thermal stability, excellent solubility, and good transparency. The fluorescent properties of D1 derivatives have been studied by Bu et al. [22].

To explore the influence of $\pi$-conjugated linkers on linear and NLO properties of the non planar derivatives D1D3 and planar derivatives D4-D8, the mean isotropic polarizability and the total first-order hyperpolarizability were calculated employing the popular CAM-B3LYP and M06-2X functionals in combination with the standard $6-311++\mathrm{G}(\mathrm{d}, \mathrm{p})$ basis set. The dipole moment and polarizability components are collected in Table 3 and the components of first hyperpolarisability as well as $\beta_{\text {tot }}$ and $\mu \beta$ values are given in Table 4 . Figure 3 shows that the CAM-B3LYP and M06-2X functionals give comparable values of ground state dipole moments and polarizabilities. The computed dipole moment values of D1-D8 range from 3.89(3.92) to 5.58(5.62) at the CAM-B3LYP(M06-2X) level as shown in Table 3. We note that the designed chromophore D8 is characterized by the highest value of the total dipole moment. Table 3 also shows that the main component, $\alpha_{x x}$, and the mean polarizability, $\langle\alpha\rangle$, significantly increase with the size of $\pi$-conjugated spacer in the following order: D2 $<$ D1 $<$ D4 $<$ D3 $<$ D6 $<$ D5 $<$ D $7<$ D8.

\footnotetext{
$<$ Insert Table 3 here $>$

$<$ Insert Table 4 here $>$

$<$ Insert Figure 3 here $>$
} 
Table 4 recapitulates the calculated values of the components of first hyperpolarizability $(\beta)$ and the molecular hyperpolarizability $(\mu \beta)$ and the following order of $\beta_{\text {tot }}$ has been obtained: D2 $<$ D1 $<$ D4 $<$ D6 $<$ D3 $<$ D5 $<$ $\mathrm{D} 8<\mathrm{D} 7$, indicating that the designed derivatives $\mathrm{D} 7$ and $\mathrm{D} 8$ are characterized by the highest NLO response. Literature suggests that in general, molecules with small HOMO-LUMO energy gap show large hyperpolarizability values [15]. This trend is correctly reproduced for all derivatives except for D6. As observed in Table 4 , the highest $\beta_{\text {tot }}$ calculated value is obtained for molecules with the extended $\pi$-conjugated systems.

It turns out from Table 4, that the NLO response of D1-D8 is characterized by one dominant hyperpolarisability component, $\beta_{\mathrm{x}}$. We note that $\beta_{\mathrm{x}}$ is the only significantly changing component and it is at least one order of magnitude greater than the other components. This means that, along the $\mathrm{x}$ axis, the CT excitation is basically unidirectional. On the other hand, A- $\pi-\mathrm{IM}-(\pi-\mathrm{D})_{2}$ derivatives displayed higher nonlinearities than that of chromophore D2 which varies in the orientation of the substituents along the imidazole ring (D- $\left.\pi-\mathrm{IM}-(\pi-\mathrm{A})_{2}\right)$. This implies that in the direction $\mathrm{C} 4 / \mathrm{C} 5 \rightarrow \mathrm{C} 2$ imidazole is more polarizable [17].

\section{UV-Vis absorption spectra}

On the basis of the optimized ground state (S0) geometries, the TD-DFT/CAM-B3LYP(M06-2X) methods were used to calculate the absorption properties and electron excitation characteristics of compounds D1-D8 in chloroform. The detailed information, namely, the maximum absorption wavelength ( $\left.\lambda_{\text {abs }}\right)$, electronic absorption energies $\left(E_{\mathrm{ge}}\right)$, oscillator strengths $\left(\mathrm{f}_{\mathrm{ge}}\right)$, transition dipole moments $\left(\mu_{\mathrm{ge}}\right)$ and the major $\mathrm{MO}$ contributions for the most relevant singlet-singlet excited states are gathered in Table 5. It is well-recognized that the CAM-BLYP and M06-2X functionals are suitable for calculating the electronic transition energies [41, 42]. Indeed, the calculated absorption wavelength $\lambda_{\text {abs }}$ value of D3 agrees well with the experimental value and the deviations are 38(30) $\mathrm{nm}$ at the CAM-B3LYP(M06-2X) level. From the calculated absorption spectra for D1-D6, the HOMO-LUMO transition configurations are predominantly responsible for the $\mathrm{S} 0 \rightarrow \mathrm{S} 1$ excitation; while the corresponding excitations for D7 and D8 correspond mainly to the HOMO $\rightarrow$ LUMO and HOMO$1 \rightarrow$ LUMO. The absorption maxima are then assigned to the ICT transition. As shown in Table 5, the spectroscopic properties of the planar derivatives D4-D8 are interesting in a way that the absorption maxima are red-shifted compared to the non planar triarylimidazoles D1-D3. For instance, when passing from D1 to D4, there is a significant bathochromic shift of $\lambda_{\mathrm{abs}}$ and an increase of the hyperpolarizability. This can be ascribed to the enhancement of $\pi$-conjugation due to molecule planarization in D4. Contrariwise, when passing from D1 to D2, there is a slight blue-shift and the hyperpolarizability is reduced (see Tables 4-5). Table 5 also shows that 
extension of the conjugation length by addition of polarizable units produces a significant red-shift in the absorption spectra of these molecules. Indeed, one can find that the $\lambda_{\text {abs }}$ of D3-D8 show bathochromic shifts compared to their parent compound D1. The TD-CAM-B3LYP(M06-2X) $\lambda_{\text {abs }}$ of D3-D8 have bathochromic shifts of 1(11), 17(20), 32(40), 5(14), 44(55) and 42(54) nm compared with that of the parent compound D1, respectively (Figure 4). So, when the spacer becomes longer, the delocalizability of the central chain is improved by adding $\pi$-electrons which allows more efficient CT and accordingly larger $\beta_{\text {tot }}$ values. It is also found that the oscillator strengths and consequently the coefficient of the absorption maximum in D7 and D8 compounds are significantly larger than that in D1. For an electronic transition, the oscillator strength is proportional to the transition moment [50]. In general, greater oscillator strength corresponds to a larger coefficient of experimental absorption or a stronger intensity of fluorescence. This indicates that D7 and D8 show larger absorption intensity than that of D1. It can be concluded that extension of $\pi$-conjugated linker enhances the absorption strength.

\section{$<$ Insert Table 5 here $>$ \\ $<$ Insert Figure 4 here $>$}

The molecular (non)linear optical properties are closely related to the features of charge transfer (CT). We used the two state model (TSM) proposed by Oudar and Chemla [40] to gain further insight into optical response of the studied compounds. According to this model, the $\alpha_{\mathrm{CT}}$ magnitude is proportional to the transition dipole moment square and inversely proportional to the excitation energy while the $\beta_{\mathrm{CT}}$ magnitude is proportional to the product of the transition dipole moment and the oscillating strength and inversely proportional to the transition energy cube [51].

$$
\begin{aligned}
& \alpha_{C T} \propto \frac{\mu_{g e}^{2}}{E_{g e}} \\
& \beta_{C T} \propto \frac{\mu_{g e} \cdot f_{g e}}{E_{g e}^{3}}
\end{aligned}
$$

where $f_{\mathrm{ge}}$ denotes the oscillator strength of the transition from the ground state to the excited state, $\mu_{\mathrm{ge}}$ is the transition dipole moment, and $E_{\mathrm{ge}}$ represents the electron absorption energy. 
The calculated values of $\alpha_{\mathrm{CT}}$ and $\beta_{\mathrm{CT}}$ are tabulated in Table 4. As we can see in Figure $5,<\alpha>$ values are well correlated to the quantity $\frac{\mu_{g e}^{2}}{E_{g e}}$ suggested by the two-state model. D8 has the largest $\mu_{g e}$ and the smallest Ege, so its $\alpha_{\mathrm{CT}}$ value is the largest, indicating that D8 has the strongest linear optical properties.

$<$ Insert Figure 5 here $>$

As seen in Figure 6, as a whole, the $\beta_{\mathrm{CT}}$ values qualitatively reproduce the general trend of the static first-

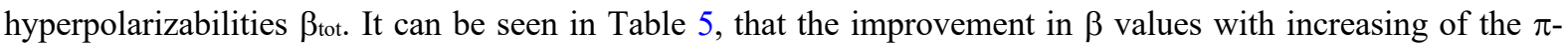
spacer can be attributed to the increasing of $\mu_{\mathrm{ge} .} f_{\mathrm{ge}}$ and decreasing $E_{\mathrm{ge}}$ values. The results show that the $\frac{\mu_{g e} \cdot f_{g e}}{E_{g e}^{3}}$ values of the studied chromophores follow the trend: D2 $<$ D1 $<$ D $4<$ D6 $<$ D3 $<$ D $5<$ D $7<$ D8 which agrees with the $\beta_{\text {tot }}$ values (Figure 6). This consistency implies that the crucial states we have noted before (Table 4) make major contributions to the $\beta$ values. Therefore, the large $\beta$ value of compound D8 can be primarily ascribed to the lower electronic absorption energy paired with stronger oscillator strength, and their strong CT characteristic. However, the smallest CAM-B3LY/M06-2X value of $\beta$ (7859.79/7769.57 a.u.) for compound D2 can be rationalized by the collective effects of its largest electronic absorption energy (3.65/3.66 eV), almost weakest oscillator strength (0.63/0.60 a.u.), and smallest transition dipole moment (2.66/2.59 a.u.).

$<$ Insert Figure 6 here $>$

In order to study the electron-transition process responsible for large NLO responses and illuminate the origins of absorption peaks, we calculated the difference in total electron density (EDD) [52] between the ground (S0) and first excited state (S1), at the CAM-B3LYP/6-31++G(d,p) level using time-dependent DFT calculations in a chloroform solvent. To visualize the charge transfer character when we changed the $\pi$-linker of the chromophores, we have plotted the electronic difference density maps (EDDMs) in Figure 1. The cyan color specifies electron density deficiency, while the purple color specifies increased electron density. Electronic distributions and localizations of the first singlet excite state visualized using the EDD maps show that the maximum absorption of all chromophores is mainly due to intramolecular electron transitions from the donor to the acceptor. For instance, the charge transfer in D4 is more pronounced than in D1, leading to a higher hyperpolarizability. 


\section{UV-Vis fluorescence spectra and Stokes shifts}

One well-known characteristic of fluorescence is that emissions usually occur at longer wavelengths than absorption wavelengths [53]. Such a process, known as Stokes shift, shows the loss of energy between absorption and emission. The fluorophore is pumped to the n-order excited state $(\mathrm{Sn})$ upon absorption, followed by rapid relaxation to the first excited state ( $\mathrm{S} 1$, internal conversion), then decaying back to the ground state ( $\mathrm{S} 0$, fluorescence). During internal conversion, loss of excitation energy results in red-shifted emissions (versus absorption) and thus, Stokes shifts. Self-absorption is a common fluorescence quenching mechanism that comes from the overlap between the spectrum of absorption and emission. The broad overlap between the absorption and emission spectra gives them a small Stokes shift for most fluorescent materials, which caused reabsorption by the fluorophore itself. Consequently, expanding their Stokes shifts, which reduces the spectral overlap of absorption and fluorophore emission, is the key for minimizing the self-absorption of the fluorophores and enhancing their efficiency effectively. To minimize reabsorption of emitted photons, Stokes shifts, typically over $80 \mathrm{~nm}$, are desirable [54]. For practical application, the large Stokes shift is advantageous as it can reduce self-quenching resulting from molecular self-absorption.

TD-CAM-B3LYP and TD-M06-2X calculations in chloroform were performed for predicting the fluorescence spectra of compounds D1-D8 using TD-CAM-B3LYP singlet excited state geometries. The fluorescence transition energies $E_{\text {flu }}$, fluorescence wavelengths $\lambda_{\text {flu }}$, main MO assignments, and the oscillator strength $f$ for the lowest singlet excited state are listed in Table 6. Pokladko-kowar et al. [28] have investigated the fluorescent properties of the commercial dye D3 and its derivatives. The calculated emission $\lambda_{\text {flu }}$ wavelength values of D3, 545(557) $\mathrm{nm}$ are in good agreement with experimental result, $535 \mathrm{~nm}$. It can be seen from the results that for fluorescence spectra, the LUMO $\leftarrow$ HOMO excitation plays a dominant role for D1-D7, whereas the fluorescence peak of $\mathrm{D} 8$ originates mainly from $\mathrm{LUMO} \leftarrow \mathrm{HOMO}$ and $\mathrm{LUMO} \leftarrow \mathrm{HOMO}-1$ excitations. The study of size and electronic nature of conjugated $\pi$-system effects suggests that the $\lambda_{\text {flu }}$ values of D3-D8 show bathochromic shifts of 13(21), 12(11), 49(54), 38(46), 104(111) and 73(82) nm as compared with that of the parent compound D1. The fluorescence color can be tuned from cyan $\left(\lambda_{\mathrm{flu}}=544 / 547 \mathrm{~nm}\right)$ to orange $\left(\lambda_{\mathrm{flu}}=636 / 647\right.$ nm). Furthermore, as shown in Table 6, the $f$ values of D3-D8 are larger than that of D1, showing stronger fluorescence intensity. On the other hand, we can also note that relatively high values of Stokes shift $(>100 \mathrm{~nm})$ are obtained from all chromophores: D1 (128/134 nm), D2 (169/172 nm), D3 (140/144 nm), D4 (123/125 nm), D5 (145/148 nm), D6 (161/166 nm), D7 (188/190 nm) and D8 (159/162) nm (Table 6). Rather broad Stokes shifts, as shown in literature, typically suggest an extensive structural reorganization in the excited state [55]. A 
plot of trends in Stokes shifts and singlet excited state dipole moment of D1-D8 is showed in Figure 7. The increase in excited state dipole moment and consequently geometric distortion are found to be responsible for the increased Stokes shifts [56]. The change in geometry in excited state suggests that electronic delocalization along the $\pi$-spacer is improved. In conclusion, the obtained results suggest that designed fluorophores D4-D8 can be considered as important building blocks used in OLED devices such as light-emission layers. This is attributed to their strong fluorescent intensity and their high Stokes shift.

$<$ Insert Table 6 here $>$
$<$ Insert Figure 7 here $>$

\section{OLED properties}

The efficiency of an OLED is determined by charge injection and transport, charge carrier balance, radiative decay of excitons, and light extraction. Charge transport is, however, one of the most important properties of OLED performance [57]. In high-performance OLED materials, the charge transport properties play a crucial role. The Marcus theory [58] can explain the rate of charge transfer by the following equation:

$$
k=\left(\frac{V^{2}}{h}\right)\left(\frac{\pi}{\lambda_{h / e} k_{B} T}\right)^{1 / 2} \exp \left(-\frac{\lambda_{h / e}}{4 k_{B} T}\right)
$$

where $\mathrm{T}$ is the temperature, the Boltzmann constant is $\mathrm{k}_{\mathrm{B}}$ and $\mathrm{V}$ is the element of the electronic coupling matrix (transfer integral) between the two adjacent species, largely determined by orbital overlap. The internal reorganization energy of the hole/electron $\left(\lambda_{\mathrm{h} / \mathrm{e}}\right)$ due to geometric relaxation accompanying charge transfer refers to the system's energy change and it is induced by structural relaxation after electron gain or loss and obtained by ignoring any relaxation and changes in the environment. Low reorganization energy is useful for transport charges. Pursuant to Eq. (6), it is obvious that the energy of reorganization should be low enough to achieve greater mobility of the hole/electron. The internal reorganization energy is commonly used to determine the rate of charge transport [59] as the key factor and can be calculated using equation (7) [60]:

$\lambda_{\mathrm{h} / \mathrm{e}}=\left(\mathrm{E}_{0}^{ \pm}-\mathrm{E}_{ \pm}\right)+\left(\mathrm{E}_{ \pm}^{0}-\mathrm{E}_{0}\right)$

where $\mathrm{E}_{0}^{+}\left(\mathrm{E}_{0}^{-}\right)$and $\mathrm{E}_{+}^{0}\left(\mathrm{E}_{-}^{0}\right)$ are the charged energy on neutral states and neutral energy used cation (anion) states, respectively; $\mathrm{E}_{+}\left(\mathrm{E}_{-}\right)$and $\mathrm{E}_{0}$ are energies for cation (anion) states and neutral state, respectively.

Listed in Table 7, the reorganization energies for electron $\left(\lambda_{\mathrm{e}}\right)$ and hole $\left(\lambda_{\mathrm{h}}\right)$ of D1-D8 molecules were predicted from the single point energy at the CAM-B3LYP/6-31G(d,p) level based on the CAM-B3LYP/6- 
$31 \mathrm{G}(\mathrm{d}, \mathrm{p})$ optimized neutral, cationic, and anionic geometries. Hole reorganization energy $\left(\lambda_{\mathrm{h}}\right)$ and electron reorganization energy $\left(\lambda_{\mathrm{e}}\right)$ were used to evaluate charge transport abilities of dyes D1-D8. The results displayed in Table 7 show that the calculated $\lambda_{\mathrm{e}}$ values of D1-D8 $(0.465-0.625 \mathrm{eV})$ are smaller than that of tris $(8-$ hydroxyquinolinato)aluminium (III) (Alq3) $\left(\lambda_{\mathrm{e}}=0.702 \mathrm{eV}\right)$, which is a typical electron transport material (ETM) [59]. It implies that the D1-D8 are predicted to be better ETMs than Alq3. On the other hand, the calculated $\lambda_{\mathrm{h}}$ values of D1-D8 (0.378-0.536 eV) are smaller than that of N,N'-diphenyl-N,N'-bis(3-methlphenyl)-(1,1'biphenyl)-4,4'-diamine (TPD) $\left(\lambda_{\mathrm{h}}=0.578 \mathrm{eV}\right)$, which is a typical hole transport material (HTM) [61]. It indicates that D1-D8 are predicted to be better HTMs than TPD. Table 7 also shows that the $\lambda_{\mathrm{e}}$ values are comparatively larger than the $\lambda_{\mathrm{h}}$ values for all the studied compounds, indicating that the hole transport performance of these dyes is better than their electron transport ability. Among the studied derivatives, D2 is found to have the poor hole transfer ability since it is characterized by the highest $\lambda_{\mathrm{h}}$ value, whereas D1 is found to have the poor electron transfer ability since it is characterized by the highest $\lambda_{\mathrm{e}}$ value. The nearly identical $\lambda_{\mathrm{h}}$ values of D5 and D6 indicate that they may have the comparable hole transfer abilities. We also found that the energy differences $(\Delta \lambda)$ between $\lambda_{\mathrm{h}}$ and $\lambda_{\mathrm{e}}$ for the designed $\mathrm{D} 2$ and $\mathrm{D} 7$ (0.06 and $0.07 \mathrm{eV}$, respectively) are smaller than those for D1, D4, D5, D6 and D8 (0.11 eV; $0.23 \mathrm{eV} ; 0.15 \mathrm{eV}$ and $0.19 \mathrm{eV}$ respectively). This indicates that D2 and D7 have good charge transport balance performance in comparison of the other derivatives, which makes these molecules potentially applicable as active emitting layers for OLEDs. The results summarized in Table 7 also show that the size of the $\pi$-conjugated bridge has significant effect on the reorganization energy for both electrons and holes. Indeed, all studied compounds D1-D8 exhibit $\pi$-character that spreads over the entire molecule resulting in delocalization, demonstrating their efficient charge transfer ability and the following sequence orders are obtained.

D2 $<$ D1 $<$ D3 $<$ D7 $\sim$ D4 $<$ D6 $<$ D5 $<$ D8 (for hole transport ability).

$\mathrm{D} 1<\mathrm{D} 4<\mathrm{D} 2<\mathrm{D} 6<\mathrm{D} 8<\mathrm{D} 5<\mathrm{D} 3<\mathrm{D} 7 \quad$ (for electron transport ability)

Consequently, D7 is predicted as the best electron transport material; whereas D8 is predicted as the best hole transport material.

$<$ Insert Table 7 here $>$

Adiabatic ionization potential (AIP) and electron affinity (EA), which are both closely related to HOMO and LUMO, respectively, must be measured in order to determine the energy barrier for hole and electron injections. A smaller AIP value means easier hole injection ability for OLED materials, while a larger 
EA value facilitates electron injection [11, 82, 83]. For D1-D8, the calculated adiabatic IP and EA are listed in Table 7. For the creation of a hole in D7, a lower IP value is beneficial, while a higher EA denotes a greater capacity for charge injection in D8.

Stability is a valuable criterion for determining the quality of devices for charge transport and luminescent materials and constitutes an important index for their use. To estimate the stability of charge transport materials (CTMs) [6], the absolute chemical hardness $(\eta)$, which can be defined as the resistance of the chemical system to exchange electronic charge with the environment [64], can be used. The absolute hardness, $\eta$, is expressed in the context of the conceptual DFT by the following equation [65]:

$\eta=\frac{A I P-E A}{2}$

where

$A I P=E_{+}^{+}-E_{0}^{0}$

$E A=E_{0}^{0}-E_{-}^{-}$

The $\eta$ values of investigated molecules were calculated and shown in Table 7 . It is evident from the inspection of Table 7 that the $\eta$ values of derivatives D1-D2, D4-D8 are almost equal to that of the synthesized D3. It can be assumed that the designed YCTCs are as stable as the D3 commercial dye and can be regarded as promising materials for transport of charges. This also means that the stability of these molecules is not greatly affected by the various kinds of $\pi$-linkers.

\section{Conclusions}

In this paper, we studied a series of imidazole-based Y-shaped charge transfer chromophores, D1-D8, in order to explore them as attracting organic materials suitable for NLO and OLED devices. DFT and TD-DFT calculations were performed to provide a detailed understanding of (non)linear, electronic, fluorescent, and charge transport properties of D-series. The obtained results show that CAM-B3LYP and M06-2X functionals give comparable values of (non)linear optical properties. The present study reveals that planarity of the chromophore, extension of conjugation and modification of electronic nature of the $\pi$-spacer by addition of easily polarizable units increase the nonlinearity and produce a significant red shift in the absorption spectra. Interestingly, the $\alpha_{\mathrm{CT}}$ and $\beta_{\mathrm{CT}}$ values, estimated using the two-state model, are well correlated to $<\alpha>$ and $\beta_{\text {tot }}$ 
values, respectively. Further, the larger hyperpolarizability together with higher excited state absor-ption coefficient values for D7 and D8 suggests a potential use of these molecules in the design of NLO devices.

From the calculated fluorescence spectra of compounds D1-D8, high Stokes Shift values $(>100 \mathrm{~nm})$ are obtained for all chromophores, indicating an extensive structural reorganization in the excited state and strong fluorescent intensity is predicted. Particularly, among the D-series, the designed fluorophores D4-D8 can be considered as important building blocks used in OLED devices such as light-emission layers. The low values of reorganization energies reveal that compounds D1-D8, particularly D8, can be considered as effective holetransporting materials. Calculated hardness values show that the designed derivatives exhibit comparable resistance to charge exchange compared to the commercial dye D3. We believe that the present contribution may offer some guidance for the design and synthesis of novel imidazole-based YCTCs with promising NLO, fluorescent and OLED properties.

\section{Electronic Supplementary Material}

Optimized cartesian coordinates of the studied chromophores D1-D8 are available in electronic supporting information file.

\section{Declarations}

Funding: N/A

Conflict of interest: The authors declare that they have no conflicts of interest.

Data availability: N/A

Availability of data and material: N/A

Code availability: N/A

Ethics approval: N/A

Consent to participate: N/A

Consent for publication : N/A

Authors' contributions: 
L. Kara Zaitri: Investigation, Writing-review and editing

S. M. Mekelleche : Conceptualization, methodology, Writing-review and editing

\section{Acknowledgments}

The authors gratefully acknowledge the University of Tlemcen for technical support. 


\section{References}

1. Bredas JL, Adant C, Tackx P, et al (1994) Third-Order Nonlinear Optical Response in Organic Materials: Theoretical and Experimental Aspects. Chem Rev 94:243-278. https://doi.org/10.1021/cr00025a008

2. Liu J, Yang Y, Liu X, Zhen Z (2015) Physical attachment of NLO chromophores to polymers for great improvement of long-term stability. Mater Lett 142:87-89. https://doi.org/10.1016/j.matlet.2014.11.146

3. Thakare SS, Sreenath MC, Chitrambalam S, et al (2017) Non-linear optical study of BODIPYbenzimidazole conjugate by solvatochromic, Z-scan and theoretical methods. Opt Mater 64:453-460. https://doi.org/10.1016/j.optmat.2017.01.020

4. Reineke S, Lindner F, Schwartz G, et al (2009) White organic light-emitting diodes with fluorescent tube efficiency. Nature 459:234-238. https://doi.org/10.1038/nature08003

5. Jungsuttiwong S, Tarsang R, Sudyoadsuk T, et al (2013) Theoretical study on novel double donor-based dyes used in high efficient dye-sensitized solar cells: The application of TDDFT study to the electron injection process. Org Electron 14:711-722. https://doi.org/10.1016/j.orgel.2012.12.018

6. Sun Z-Z, Xu Y-L, Zhu R, Liu H-Y (2018) How to stabilize the HOMO levels and to improve the charge transport properties of hole-transporting materials? Probing the effects of molecular symmetry. Org Electron 63:86-92. https://doi.org/10.1016/j.orgel.2018.09.013

7. Coropceanu V, Cornil J, Demetrio A. da Silva Filho, et al (2007) Charge Transport in Organic Semiconductors. https://pubs.acs.org/doi/pdf/10.1021/cr050140x

8. Ostroverkhova O (2016) Organic Optoelectronic Materials: Mechanisms and Applications. https://pubs.acs.org/doi/pdf/10.1021/acs.chemrev.6b00127

9. Janjua MRSA (2012) Quantum Mechanical Design of Efficient Second-Order Nonlinear Optical Materials Based on Heteroaromatic Imido-Substituted Hexamolybdates: First Theoretical Framework of POM-Based Heterocyclic Aromatic Rings. https://pubs.acs.org/doi/pdf/10.1021/ic3002652

10. Chai W, Jin R (2016) Theoretical investigations into optical and charge transfer properties of donoracceptor 1,8-naphthalimide derivatives as possible organic light-emitting materials. J Mol Struct 1103:177-182. https://doi.org/10.1016/j.molstruc.2015.09.023

11. Reeta Felscia U, Rajkumar BJM, Briget Mary M (2018) Charge transport properties of pyrene and its derivatives: optoelectronic and nonlinear optical applications. J Mater Sci 53:15213-15225. https://doi.org/10.1007/s10853-018-2690-9

12. Klikar M, Solanke P, Tydlitát J, Bureš F (2016) Alphabet-Inspired Design of (Hetero)Aromatic Push-Pull Chromophores. Chem Rec 16:1886-1905. https://doi.org/10.1002/tcr.201600032

13. Jin L, Shi S, Zhao C, et al (2021) Y-shaped organic dyes with D2- $\pi-$ A configuration as efficient cosensitizers for ruthenium-based dye sensitized solar cells. J Power Sources 481:228952. https://doi.org/10.1016/j.jpowsour.2020.228952

14. Kara Zaitri L, Mekelleche SM (2020) Nonlinear optical activity of imino-dyes with furan, thiophene or thiazole moieties as $\pi$-conjugated bridge: a computational investigation. Mol Phys 118:e1761471. https://doi.org/10.1080/00268976.2020.1761471

15. Khan MU, Khalid M, Ibrahim M, et al (2018) First Theoretical Framework of TriphenylamineDicyanovinylene-Based Nonlinear Optical Dyes: Structural Modification of $\pi$-Linkers. https://pubs.acs.org/doi/pdf/10.1021/acs.jpcc.7b12293

16. Kara Zaitri L, Mekelleche SM (2020) Computational study of linear and nonlinear optical properties of substituted thiophene imino dyes using long-range corrected hybrid DFT methods. Mol Phys 118:1618508. https://doi.org/10.1080/00268976.2019.1618508 
17. Kulhánek J, Bureš F (2012) Imidazole as a parent $\pi$-conjugated backbone in charge-transfer chromophores. Beilstein J Org Chem 8:25-49. https://doi.org/10.3762/bjoc.8.4

18. Wu W, Ye C, Wang D (2003) Design and syntheses of efficient and thermally stable diamino chromophores for NLO application. Arkivoc 2003:59-69. https://doi.org/10.3998/ark.5550190.0004.208

19. Hloušková Z, Bureš F (2017) Synthesis and properties of push-pull imidazole derivatives with application as photoredox catalysts. Arkivoc 2017:330-342. https://doi.org/10.24820/ark.5550190.p010.071

20. Kulhánek J, Bureš F, Mikysek T, et al (2011) Imidazole as a central $\pi$-linkage in Y-shaped push-pull chromophores. Dyes Pigments 90:48-55. https://doi.org/10.1016/j.dyepig.2010.11.004

21. Miller RD, Lee VY, Moylan CR (2002) Substituted Azole Derivatives as Nonlinear Optical Chromophores. https://pubs.acs.org/doi/pdf/10.1021/cm00043a026

22. Wu W, Zhang Z, Zhang X (2005) Design and syntheses of highly efficient and thermally-stable imidazole chromophores bearing diamino groups for nonlinear optics. J Nonlinear Opt Phys Mater 14:61-65. https://doi.org/10.1142/S0218863505002499

23. Moylan CR, Miller RD, Twieg RJ, et al (2002) Synthesis and nonlinear optical properties of donoracceptor substituted triaryl azole derivatives. https://pubs.acs.org/doi/pdf/10.1021/cm00034a021

24. Guo J-G, Cui Y-M, Lin H-X, et al (2011) New fluorene derivatives based on 3,9-dihydrofluoreno[3,2d]imidazole (FI): Characterization and influence of substituents on photoluminescence. J Photochem Photobiol Chem 219:42-49. https://doi.org/10.1016/j.jphotochem.2011.01.014

25. Parr RG, Yang W (1989) Density-Functional Theory of Atoms and Molecules New York: Oxford Univ. Press

26. Runge E, Gross EKU (1984) Density-Functional Theory for Time-Dependent Systems. Phys Rev Lett 52:997-1000. https://doi.org/10.1103/PhysRevLett.52.997

27. Santos J, Mintz EA, Zehnder O, et al (2001) New class of imidazoles incorporated with thiophenevinyl conjugation pathway for robust nonlinear optical chromophores. Tetrahedron Lett 42:805-808. https://doi.org/10.1016/S0040-4039(00)02143-2

28. Pokladko-Kowar M, Nosidlak N, Gondek E, et al (2016) Optoelectronic features of Y-shaped push-pull molecules based on imidazole. Opt Quantum Electron 48:82. https://doi.org/10.1007/s11082-015-0263-6

29. Schlegel HB (1982) Optimization of equilibrium geometries and transition structures. J Comput Chem 3:214-218. https://doi.org/10.1002/jcc.540030212

30. Hariharan PC, Pople JA (1974) Accuracy of AH n equilibrium geometries by single determinant molecular orbital theory. Mol Phys 27:209-214. https://doi.org/10.1080/00268977400100171

31. Gaussian 09, Revision A. 02 (2009), Frisch MJ, Trucks GW, Schlegel HB, Scuseria GE, Robb MA, Cheeseman JR, Scalmani G, Barone V, Mennucci B, Petersson GA, Nakatsuji H, Caricato M, Li X, Hratchian HP, Izmaylov AF, Bloino J, Zheng G., Clemente F, Sonnenberg JL, Hada M, Ehara M, Toyota K, Fukuda R, Hasegawa J, Ishida M, Nakajima T, Honda Y, Kitao O, Nakai H, Vreven T, Montgomery JA, Peralta JE, Ogliaro F, Bearpark M, Heyd JJ, Brothers E, Kudin KN, Staroverov VN, Keith T, Kobayashi R, Normand J, Raghavachari K, Rendell A, Burant JC, Iyengar SS, Tomasi J, Cossi M, Rega N, Millam JM, Klene M, Knox JE, Cross JB, Bakken V, Adamo C, Jaramillo J, Gomperts R, Stratmann RE, Yazyev O, Austin AJ, Cammi R, Pomelli C, Ochterski JW, Martin RL, Morokuma K, Zakrzewski VG, Voth GA, Salvador P, Dannenberg JJ, Dapprich S, Daniels AD, Farkas O, Foresman JB, Ortiz JV, Cioslowski J, Fox DJ, Gaussian, Inc., Wallingford CT, 2013.

32. Yanai T, Tew DP, Handy NC (2004) A new hybrid exchange-correlation functional using the Coulombattenuating method (CAM-B3LYP). Chem Phys Lett 393:51-57. https://doi.org/10.1016/j.cplett.2004.06.011 
33. Rostov IV, Amos RD, Kobayashi R, et al (2010) Studies of the Ground and Excited-State Surfaces of the Retinal Chromophore using CAM-B3LYP. https://pubs.acs.org/doi/pdf/10.1021/jp911329g

34. Pedone A (2013) Role of Solvent on Charge Transfer in 7-Aminocoumarin Dyes: New Hints from TDCAM-B3LYP and State Specific PCM Calculations. https://pubs.acs.org/doi/pdf/10.1021/ct4004349

35. Zhao Y, Truhlar DG (2011) Applications and validations of the Minnesota density functionals. Chem Phys Lett 502:1-13. https://doi.org/10.1016/j.cplett.2010.11.060

36. Tonnelé C, Champagne B, Muccioli L, Castet F (2018) Second-order nonlinear optical properties of Stenhouse photoswitches: insights from density functional theory. Phys Chem Chem Phys 20:2765827667. https://doi.org/10.1039/C8CP05843A

37. Liu Y, Yuan Y, Tian X, et al (2020) High first-hyperpolarizabilities of thiobarbituric acid derivativebased donor- $\pi$-acceptor nonlinear optical-phores: Multiple theoretical investigations of substituents and conjugated bridges effect. Int J Quantum Chem 120:e26176. https://doi.org/10.1002/qua.26176

38. Wei J, Song P, Ma F, et al (2020) Tunable linear and nonlinear optical properties of chromophores containing 3,7-(di)vinylquinoxalinone core by modification of receptors moieties. Opt Mater 99:109580. https://doi.org/10.1016/j.optmat.2019.109580

39. Lu D, Chen G, Perry JW, Goddard WA (1994) Valence-Bond Charge-Transfer Model for Nonlinear Optical Properties of Charge-Transfer Organic Molecules. J Am Chem Soc 116:10679-10685. https://doi.org/10.1021/ja00102a037

40. Oudar JL, Chemla DS (1977) Hyperpolarizabilities of the nitroanilines and their relations to the excited state dipole moment. J Chem Phys 66:2664-2668. https://doi.org/10.1063/1.434213

41. Avcı D, Tamer Ö, Atalay Y (2016) Solvatochromic effect on UV-vis absorption and fluorescence emission spectra, second- and third-order nonlinear optical properties of dicyanovinyl-substituted thienylpyrroles: DFT and TDDFT study. J Mol Liq 220:495-503. https://doi.org/10.1016/j.molliq.2016.05.023

42. Cheng X (2016) TD-M06-2X insights into the absorption and emission spectra of dichlorvos and its molecularly imprinted recognition by methacrylic acid. J Mol Model 22:282. https://doi.org/10.1007/s00894-016-3151-5

43. Tomasi J, Mennucci B, Cammi R (2005) Quantum Mechanical Continuum Solvation Models. Chem Rev 105:2999-3094. https://doi.org/10.1021/cr9904009

44. Miertuš S, Scrocco E, Tomasi J (1981) Electrostatic interaction of a solute with a continuum. A direct utilizaion of $\mathrm{AB}$ initio molecular potentials for the prevision of solvent effects. Chem Phys 55:117-129. https://doi.org/10.1016/0301-0104(81)85090-2

45. Marcano E, Squitieri E, Murgich J, Soscún H (2015) Theoretical investigation of the static (hyper)polarizabilities and reorganization energy of 4,5-dicyanoimidazole chromophore and derivatives containing benzene rings and a saturated bridge. Comput Theor Chem 1057:60-66. https://doi.org/10.1016/j.comptc.2015.01.013

46. De Oliveira MA, Duarte HA, Pernaut J-M, De Almeida WB (2000) Energy Gaps of $\alpha, \alpha^{6}$-Substituted Oligothiophenes from Semiempirical, $\mathrm{Ab}$ Initio, and Density Functional Methods. https://pubs.acs.org/doi/pdf/10.1021/jp001252p

47. Reed AE, Curtiss LA, Weinhold F (2002) Intermolecular interactions from a natural bond orbital, donoracceptor viewpoint. https://pubs.acs.org/doi/pdf/10.1021/cr00088a005

48. Weinhold F, Landis CR (2005) Valency and bonding: a natural bond orbital donor-acceptor perspective. Cambridge University Press, Cambridge, 1-760. 
49. Prasad PN, Williams DJ (1991) Introduction to nonlinear optical effects in molecules and polymers, John Wiley \& Sons, New York.

50. Weinhold F, Schleyer PVR (1998) Encyclopedia of computational chemistry. Wiley New York 3:1792.

51. Janjua MRSA, Liu C-G, Guan W, et al (2009) Prediction of Remarkably Large Second-Order Nonlinear Optical Properties of Organoimido-Substituted Hexamolybdates. J Phys Chem A 113:3576-3587. https://doi.org/10.1021/jp808707q

52. Steiner E (1982) Density-difference maps in quantum chemistry. Theor Chim Acta 60:561-572. https://doi.org/10.1007/BF00549611

53. Lakowicz JR (2013) Principles of fluorescence spectroscopy. Springer science \& business media.

54. Araneda JF, Piers WE, Heyne B, et al (2011) High Stokes Shift Anilido-Pyridine Boron Difluoride Dyes. Angew Chem Int Ed 50:12214-12217. https://doi.org/10.1002/anie.201105228

55. Kumar D, Thomas KRJ (2011) Optical properties of pyrene and anthracene containing imidazoles: Experimental and theoretical investigations. J Photochem Photobiol Chem 218:162-173. https://doi.org/10.1016/j.jphotochem.2010.12.018

56. Gawale Y, Sekar N (2018) Investigating the excited state optical properties and origin of large stokes shift in Benz[c,d]indole N-Heteroarene BF2 dyes with ab initio tools. J Photochem Photobiol B 178:472-480. https://doi.org/10.1016/j.jphotobiol.2017.12.006

57. Marcus RA (1964) Chemical and Electrochemical Electron-Transfer Theory. Annu Rev Phys Chem 15:155-196. https://doi.org/10.1146/annurev.pc.15.100164.001103

58. Marcus RA (1993) Electron transfer reactions in chemistry. Theory and experiment. Rev Mod Phys 65:599-610. https://doi.org/10.1103/RevModPhys.65.599

59. Lin BC, Cheng CP, You Z-Q, Hsu C-P (2005) Charge Transport Properties of Tris(8hydroxyquinolinato)aluminum(III): Why It Is an Electron Transporter. J Am Chem Soc 127:66-67. https://doi.org/10.1021/ja045087t

60. Stark MS (1997) Epoxidation of Alkenes by Peroxyl Radicals in the Gas Phase: Structure-Activity Relationships. J Phys Chem A 101:8296-8301. https://doi.org/10.1021/jp972054+

61. He Y, Hu Y, Chen X, et al (2013) Abnormal Temperature-Dependent Electron Transport in Hole Transport Material N,N'-Diphenyl-N,N'-bis(3- methylphenyl)-[1,1'-biphenyl]-4,4'-diamine (TPD). https://pubs.acs.org/doi/pdf/10.1021/jp401914n

62. Shang X, Wan N, Han D, Zhang G (2014) A theoretical study on the injection, transport, absorption and phosphorescence properties of heteroleptic iridium(III) complexes with different ancillary ligands. Photochem Photobiol Sci 13:574-582. https://doi.org/10.1039/C3PP50394A

63. Louis E, San-Fabián E, Díaz-García MA, et al (2017) Are Electron Affinity and Ionization Potential Intrinsic Parameters to Predict the Electron or Hole Acceptor Character of Amorphous Molecular Materials? J Phys Chem Lett 8:2445-2449. https://doi.org/10.1021/acs.jpclett.7b00681

64. Pearson RG (2002) Absolute electronegativity and absolute hardness of Lewis acids and bases. https://pubs.acs.org/doi/pdf/10.1021/ja00310a009

65. Parr RG, Pearson RG (2002) Absolute hardness: companion parameter to absolute electronegativity. https://pubs.acs.org/doi/pdf/10.1021/ja00364a005 


\section{Scheme and Figure captions}

Scheme 1a. Chemical structures of chromophores D1-D3.

Scheme 1b. Chemical structures of chromophores D4-D8.

Fig. 1 The frontier orbital plots of the HOMO and LUMO of chromophores D1-D8 in ground state. Electron density difference maps (EDDM) of compounds D1-D8. Cyan and purple colors indicate depletion and accumulation of electron density, respectively.

Fig. 2 Correlation between $\mathrm{E}^{(2)}$ (red line) and $\Delta \mathrm{E}_{\mathrm{H}-\mathrm{L}}$ (blue line) values for chromophores D1-D8.

Fig. 3 Comparison between CAM-B3LYP and M06-2X values of $<\alpha>$ and $\beta_{\text {tot. }}$.

Fig. 4 UV-Visible absorption spectra of chromophores D1-D8 in chloroform, calculated at TD-CAM-B3LYP/6$31+\mathrm{G}(\mathrm{d}, \mathrm{p})$ level and TD-M06-2X/6-31+G(d,p) levels.

Fig. 5 Correlation between $\left\langle\alpha>\right.$ values (blue line) and the corresponding $\alpha_{\mathrm{CT}}$ values (red line) for chromophores D1-D8.

Fig. 6 Correlation between the $\beta_{\text {tot }}$ values (blue line) and the corresponding $\beta_{\mathrm{CT}}$ values (red line) for chromophores D1-D8.

Fig. 7 Correlation between Stokes shifts (nm) (blue) and excited state dipole moment $\mu \mathrm{E}$ (D) (red). 

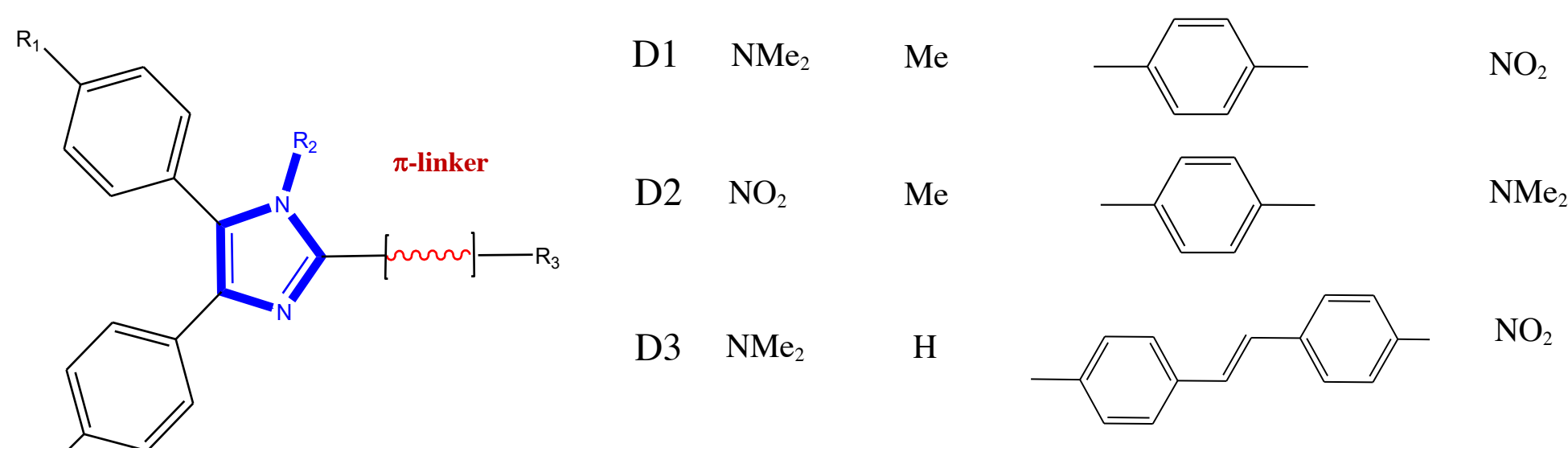

Scheme 1a. 


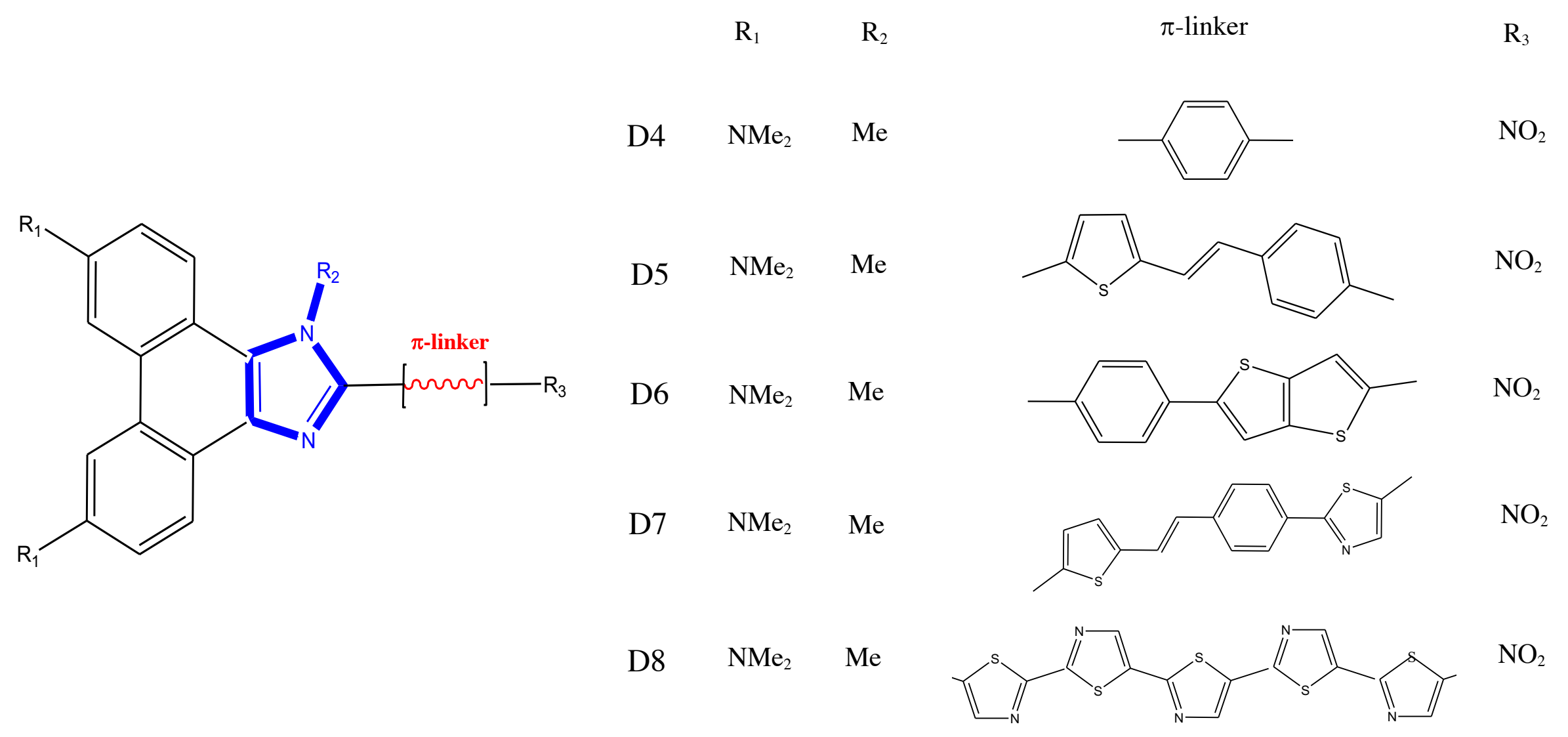

Scheme 1b. 
D1
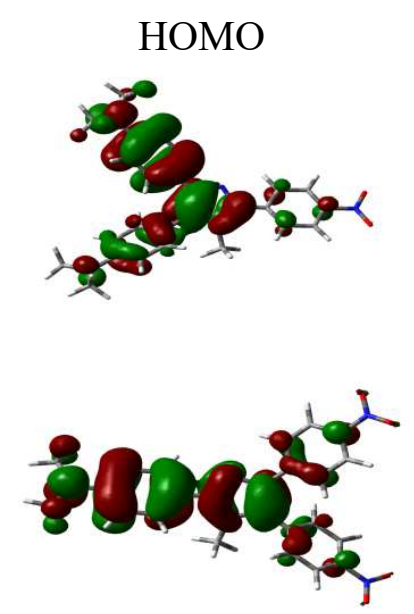

D3

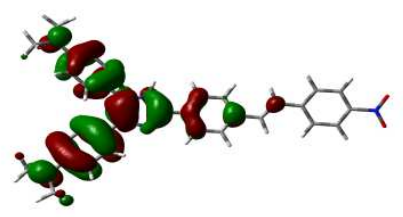

D4

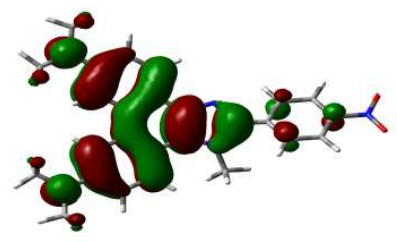

D5

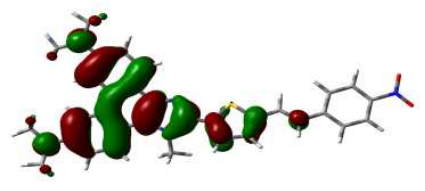

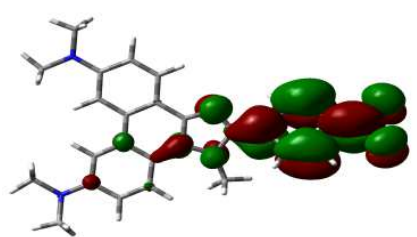
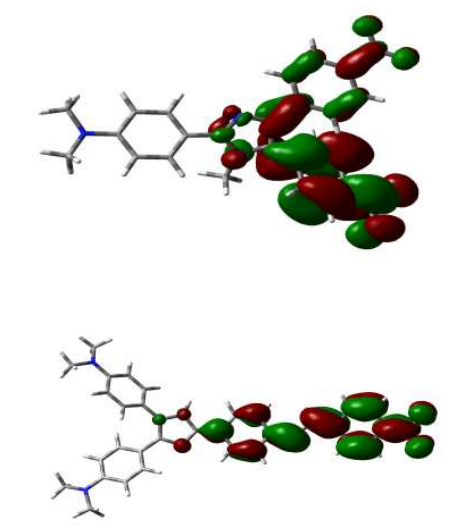

EDDM

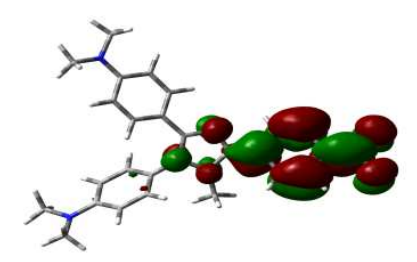

aुs

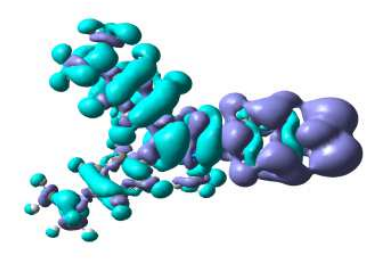

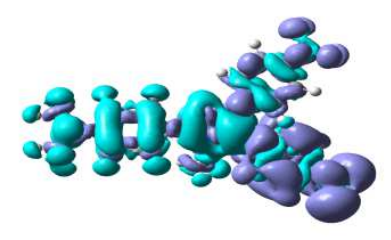
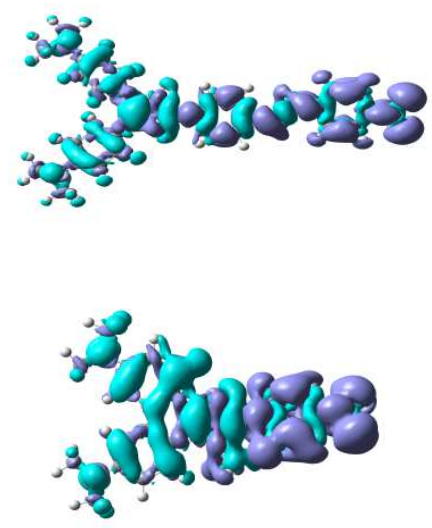

D6 कीविक्षि

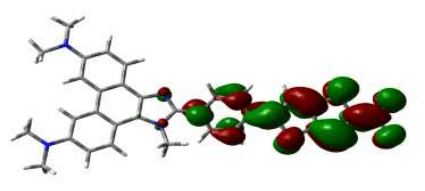

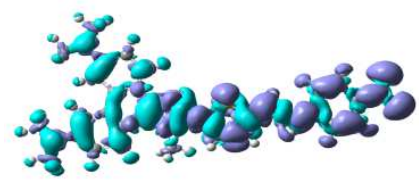

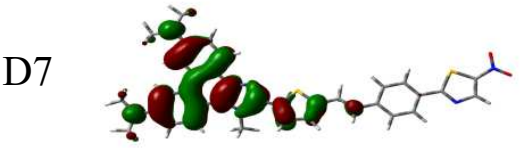

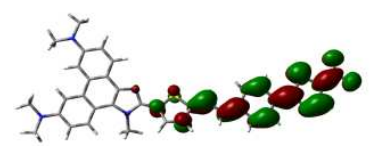

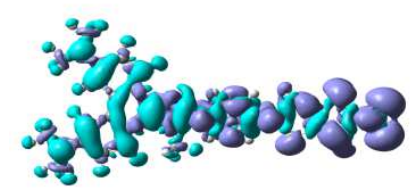

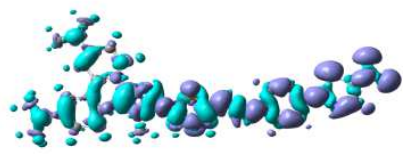

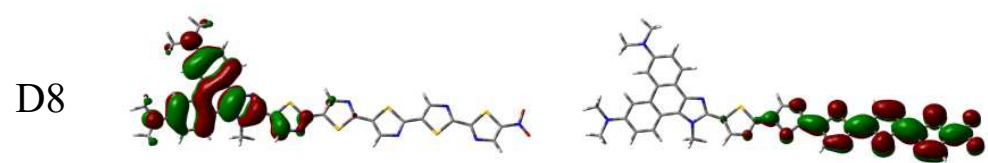

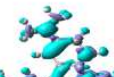
भू. 


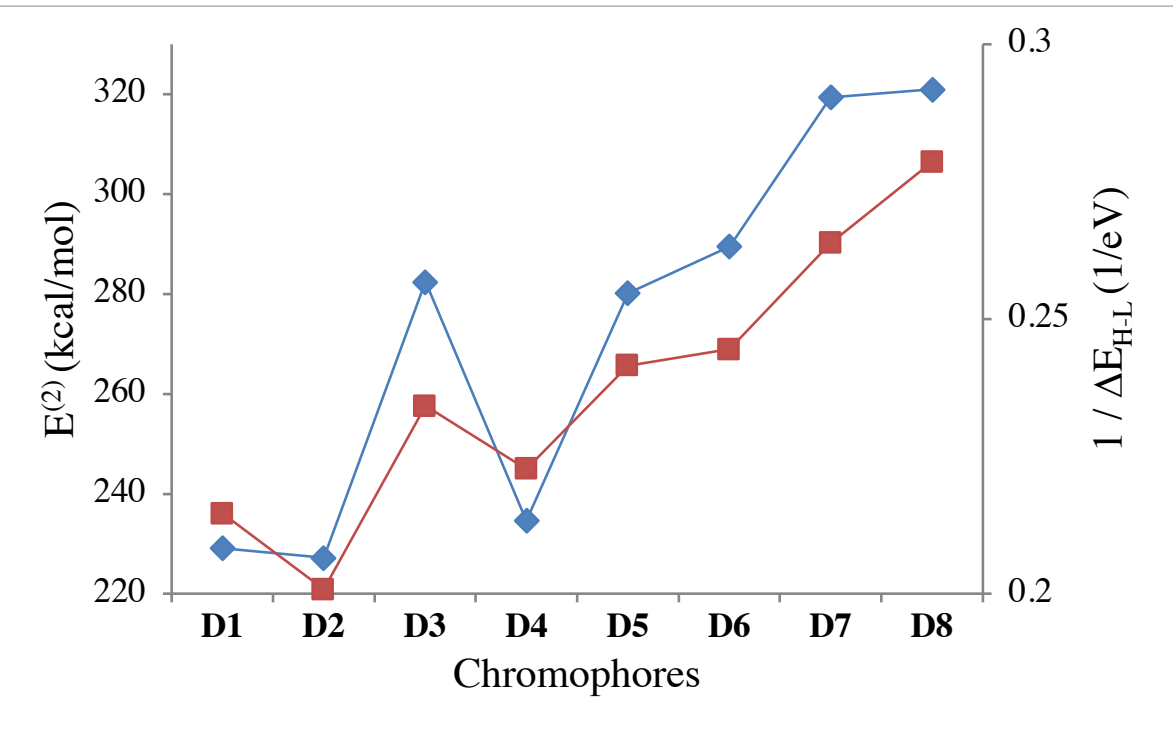

Fig. 2 

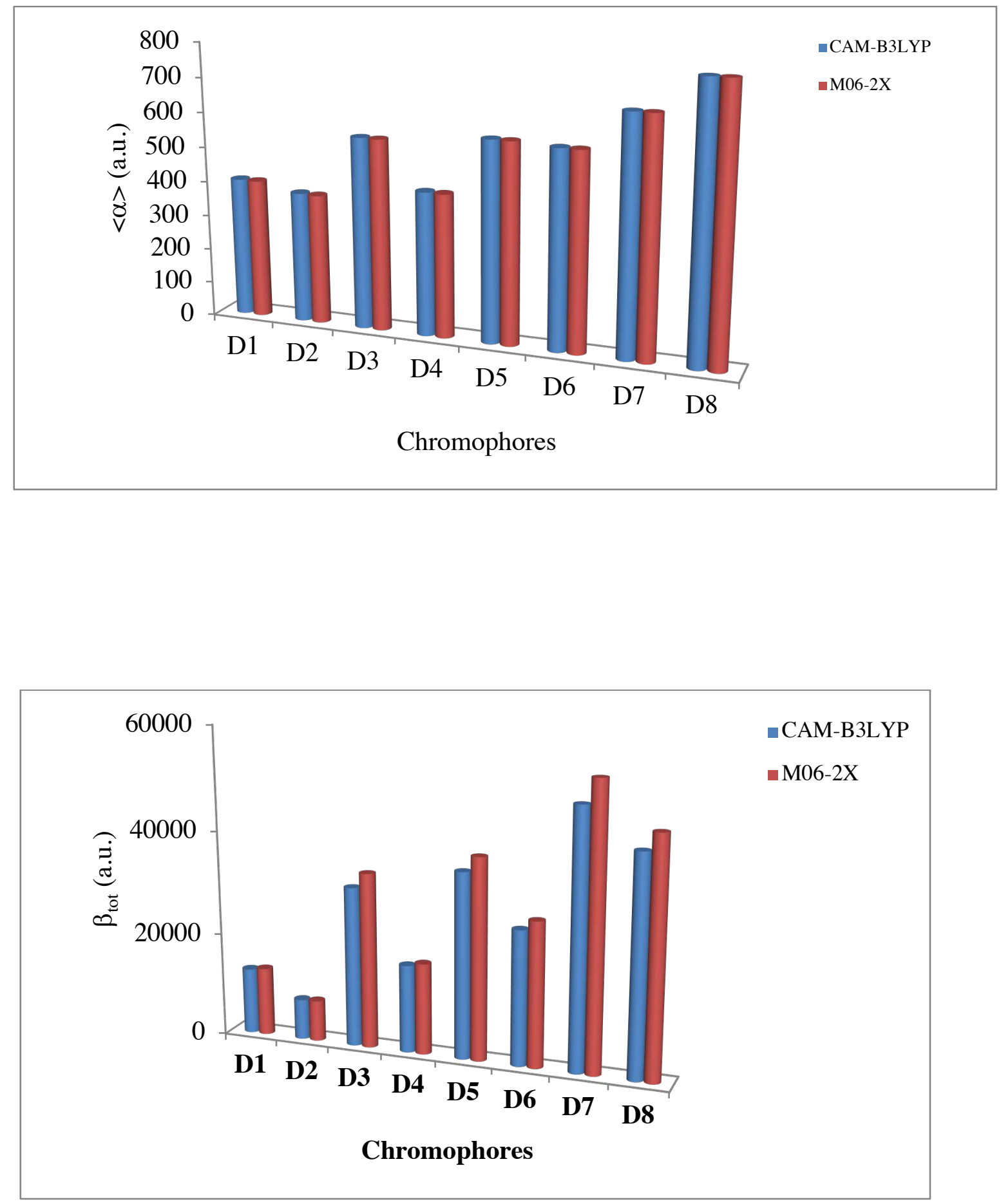

Fig. 3 

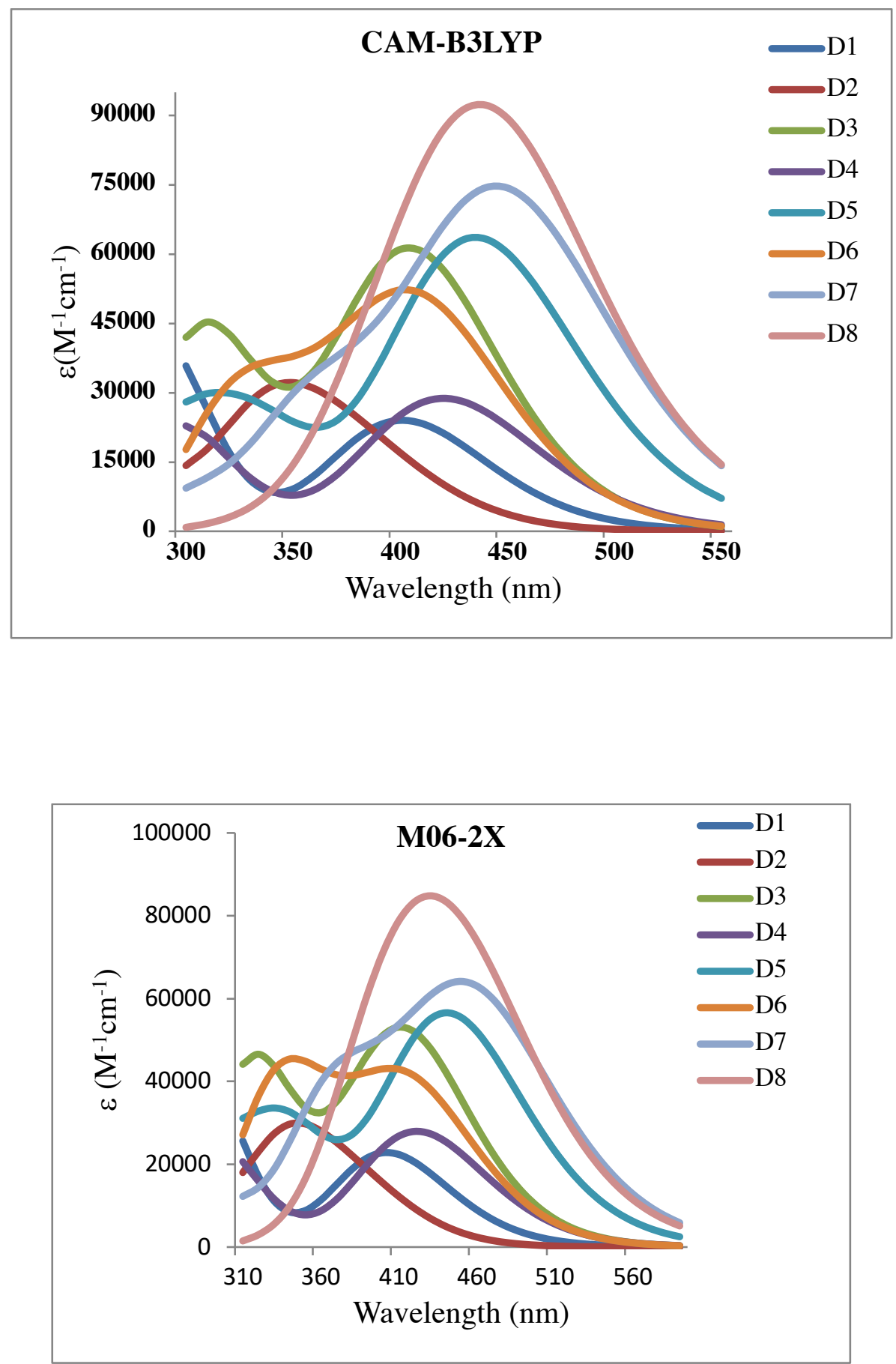

Fig. 4 

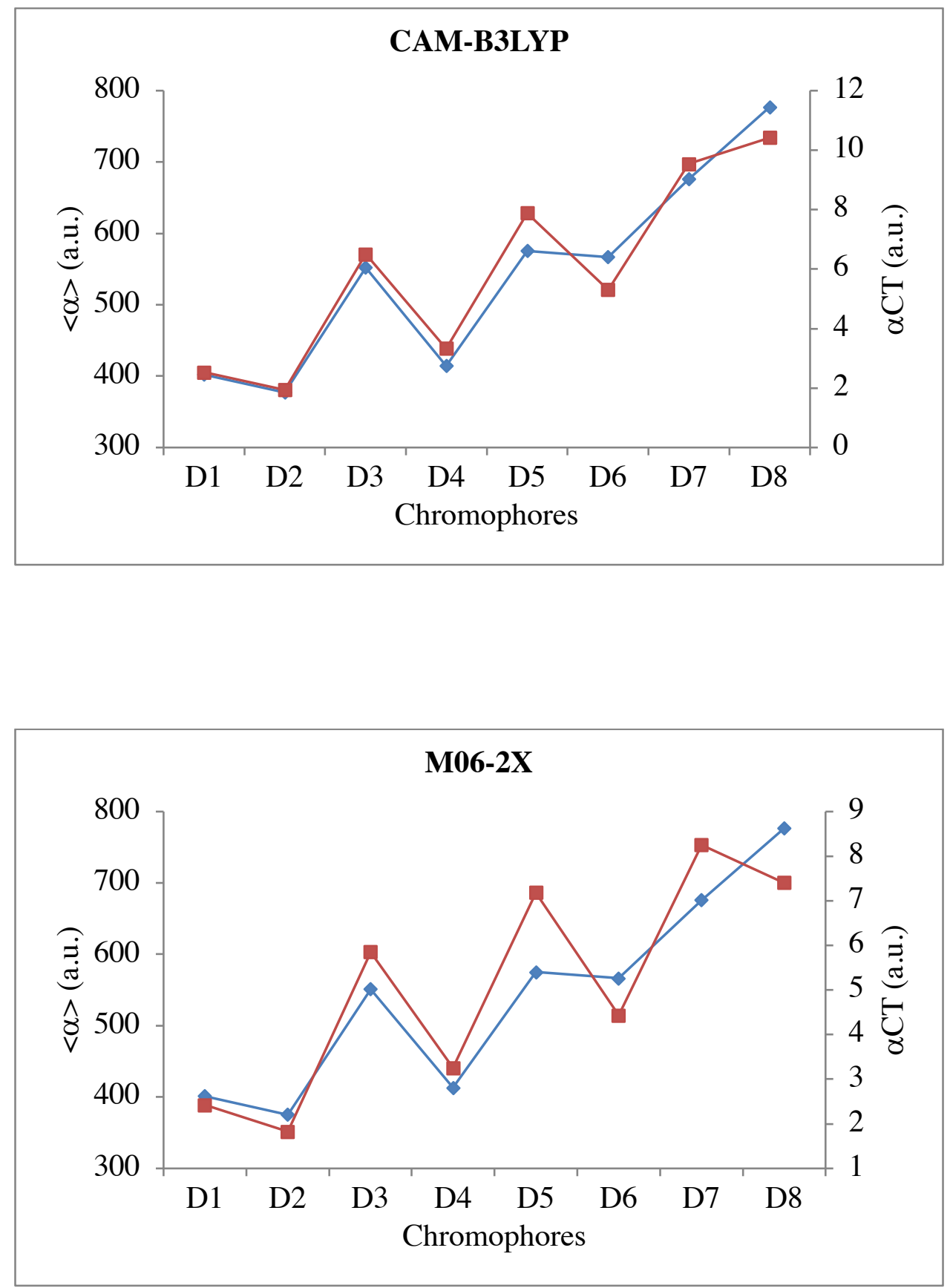

Fig. 5 

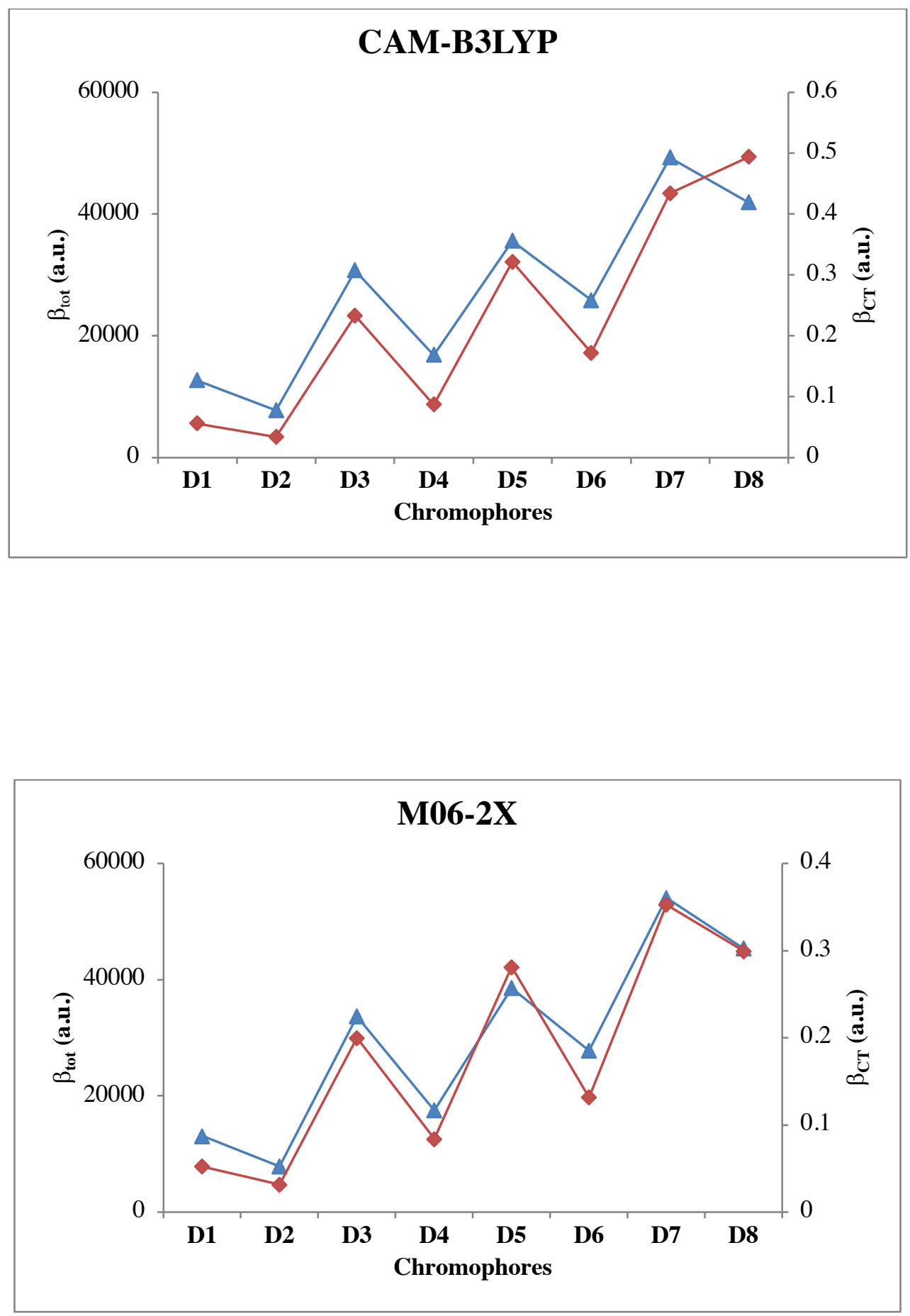

Fig. 6 

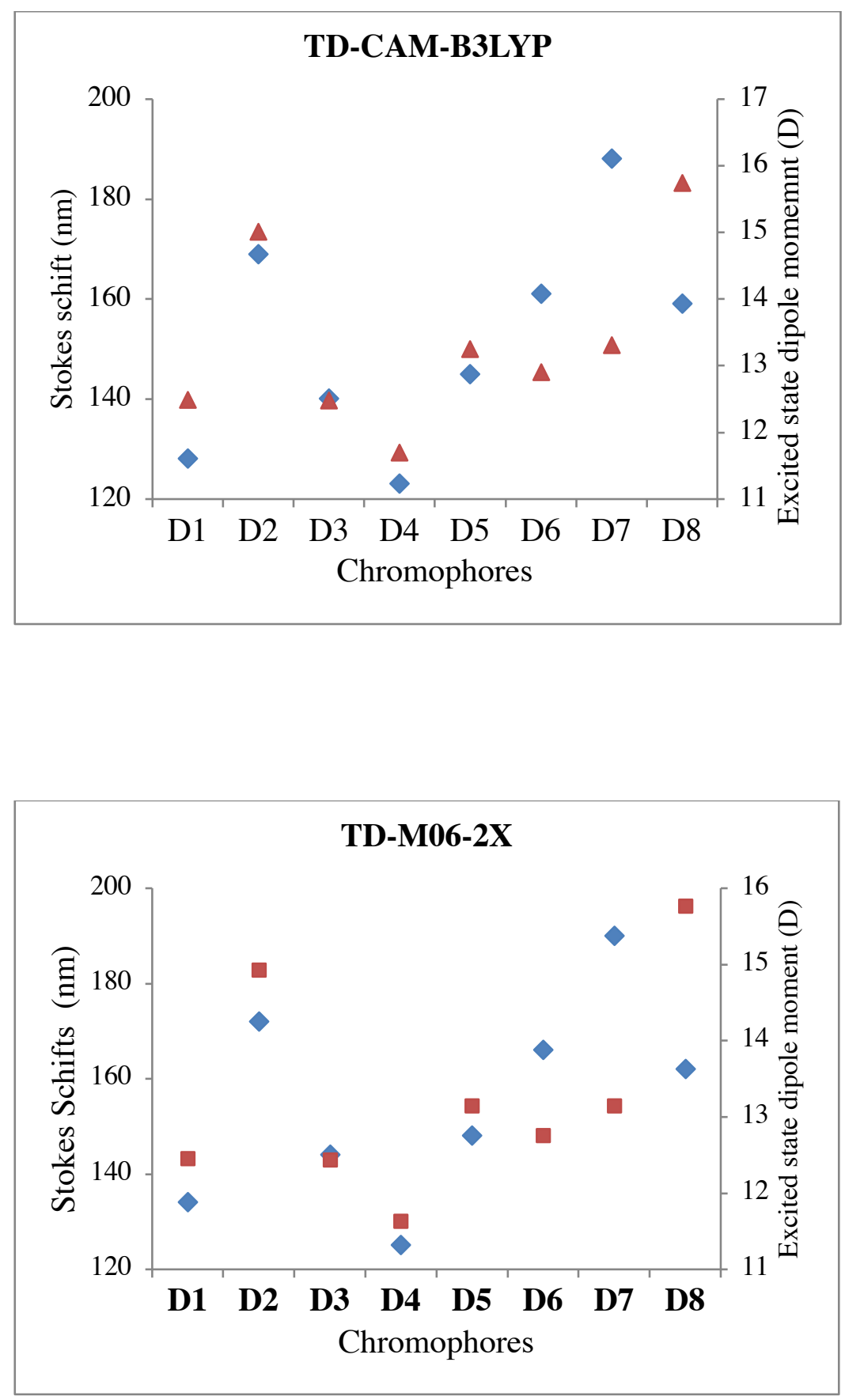

Fig. 7 


\section{Table captions}

Table 1 Highest Occupied, Lowest Unoccupied Molecular orbitals energies and energy gap HOMOLUMO (all in eV) for YCTCs D1-D8.

Table 2 Natural charges of donor, $\pi$-linker and acceptor moieties, and stabilization energies $\mathrm{E}^{(2)}$ (in kcal.mol ${ }^{-1}$ ) for YCTCs D1-D8.

Table 3 Components of dipole moment, total static dipole moment ( $\mu_{\mathrm{i}}$, in a.u.), components of polarizability and average polarizability ( $\alpha_{\mathrm{ii}}$ and $<\alpha>$, in a.u.) for YCTCs D1-D8.

Table 4 Components of first-order hyperpolarisability ( $\beta_{\mathrm{i}}$ in a.u.), and molecular hyperpolarisability $\mu \beta$ values (in $10^{-48} \mathrm{esu}$ ).

Table 5 The maximum absorption wavelengths ( $\lambda_{\max }$, in $\left.\mathrm{nm}\right)$, excitation energies $\left(\mathrm{E}_{\mathrm{ge}}\right.$, in eV), oscillator strength $f_{g e}$, main MO contributions to the electronic transitions, electronic transition moment ( $\mu_{g e}$, in a.u.), $\alpha_{\mathrm{cT}}\left(\right.$ in a.u.) and $\beta_{\mathrm{CT}}($ in a.u.) of D1-D8 in chloroform.

Table 6 The UV-Vis fluorescence wavelengths $\lambda_{\text {flu }}(\mathrm{nm})$, fluorescence transition energies $\mathrm{E}_{\text {flu }}(\mathrm{eV})$, oscillator strength $f$, main MO contributions to the electronic transitions, Stokes shifts (SS, nm), and excited state dipole moment $\left(\mu_{\mathrm{E}}, \mathrm{D}\right)$ of D1-D8 in chloroform.

Table 7 Calculated molecular reorganization energy for hole $\lambda_{\mathrm{h}}$, for electron $\lambda_{\mathrm{e}}$, and $\Delta \lambda$, adiabatic ionization potential AIP, adiabatic electronic affinity EA and absolute hardness (all in eV) of D1-D8, Alq3 and TPD at the CAM-B3LYP/6-31G(d,p) level. 
Table 1

\begin{tabular}{lrcc}
\hline & HOMO & LUMO & $\Delta \mathrm{E}_{\mathrm{H}-\mathrm{L}}$ \\
\hline D1 & -6.17 & -1.52 & 4.66 \\
D2 & -6.91 & -1.93 & 4.98 \\
D3 & -6.04 & -1.77 & 4.27 \\
D4 & -6.11 & -1.61 & 4.49 \\
D5 & -5.96 & -1.82 & 4.14 \\
D6 & -6.01 & -1.92 & 4.09 \\
D7 & -5.94 & -2.14 & 3.79 \\
D8 & -6.07 & -2.48 & 3.59 \\
\hline
\end{tabular}

Table 2

\begin{tabular}{lllll}
\hline & \multicolumn{3}{c}{ NPA Charges } & \multirow{2}{*}{$\mathrm{E}^{(2)}$} \\
\cline { 2 - 4 } & Donor & $\pi$-linker & Acceptor & \\
\hline D1 & $+0,0794$ & $+0,1874$ & $-0,2667$ & 229.11 \\
D2 & $+0,0967$ & $+0,4167$ & $-0,5133$ & 227.20 \\
D3 & $+0,0599$ & $+0,2056$ & $-0,2655$ & 282.41 \\
D4 & $+0,0705$ & $+0,1927$ & $-0,2632$ & 234.66 \\
D5 & $+0,0450$ & $+0,2212$ & $-0,2663$ & 280.17 \\
D6 & $+0,0465$ & $+0,2392$ & $-0,2857$ & 289.50 \\
D7 & $+0,0209$ & $+0,2529$ & $-0,2738$ & 319.45 \\
D8 & $+0,0569$ & $+0,2013$ & $-0,2583$ & 320.99 \\
\hline
\end{tabular}


Table 3

\begin{tabular}{|c|c|c|c|c|c|c|c|c|c|c|c|c|c|c|c|c|}
\hline & \multicolumn{8}{|c|}{ CAM-B3LYP/6-311++G(d. p) } & \multicolumn{8}{|c|}{ M06-2X/6-311++G(d.p) } \\
\hline & $\mu_{\mathrm{x}}$ & $\mu_{\mathrm{y}}$ & $\mu_{\mathrm{z}}$ & $\mu_{\text {tot }}$ & $\alpha_{x x}$ & $\alpha_{y y}$ & $\alpha_{\mathrm{zz}}$ & $<\alpha>$ & $\mu_{\mathrm{x}}$ & $\mu_{\mathrm{y}}$ & $\mu_{\mathrm{z}}$ & $\mu_{\text {tot }}$ & $\alpha_{x x}$ & $\alpha_{\mathrm{yy}}$ & $\alpha_{z z}$ & $<\alpha>$ \\
\hline D1 & 3.97 & -1.48 & 0.01 & 4.24 & 564.45 & 413.15 & 229.21 & 402.27 & 4.00 & -1.47 & 0.01 & 4.26 & 563.58 & 412.88 & 227.33 & 401.26 \\
\hline $\mathrm{D} 2$ & 4.81 & -1.76 & 0.11 & 5.13 & 526.06 & 391.74 & 213.54 & 377.11 & 4.84 & -1.75 & 0.11 & 5.15 & 524.87 & 388.49 & 212.07 & 375.15 \\
\hline D3 & -4.08 & 1.44 & -0.12 & 4.33 & 897.79 & 506.22 & 252.77 & 552.26 & -4.12 & 1.43 & -0.12 & 4.36 & 899.05 & 504.33 & 250.83 & 551.40 \\
\hline D4 & -3.67 & -1.30 & 0.00 & 3.89 & 620.55 & 428.44 & 194.11 & 414.37 & -3.70 & -1.29 & 0.00 & 3.92 & 620.03 & 427.12 & 192.55 & 413.23 \\
\hline D5 & -4.08 & -1.72 & 0.27 & 4.44 & 980.64 & 513.69 & 232.35 & 575.56 & -4.12 & -1.70 & 0.27 & 4.46 & 981.93 & 512.06 & 230.65 & 574.88 \\
\hline D6 & 4.18 & -1.22 & 0.03 & 4.36 & 937.98 & 523.11 & 239.88 & 566.99 & 4.19 & -1.19 & 0.04 & 4.37 & 939.19 & 521.80 & 238.28 & 566.42 \\
\hline D7 & -4.19 & -1.73 & -0.12 & 4.53 & 1189.77 & 579.01 & 260.09 & 676.29 & -4.20 & -1.71 & -0.12 & 4.54 & 1193.19 & 576.52 & 258.20 & 675.97 \\
\hline D8 & 5.49 & -0.95 & 0.31 & 5.58 & 1407.39 & 622.89 & 300.91 & 777.06 & 5.53 & -0.94 & 0.31 & 5.62 & 1408.66 & 621.25 & 299.78 & 776.56 \\
\hline
\end{tabular}


Table 4

\begin{tabular}{|c|c|c|c|c|c|c|c|c|c|c|}
\hline & \multicolumn{5}{|c|}{ CAM-B3LYP/6-311++G(d. p) } & \multicolumn{5}{|c|}{ M06-2X/6-311++G(d.p) } \\
\hline & $\beta_{\mathrm{x}}$ & $\beta_{\mathrm{y}}$ & $\beta_{\mathrm{z}}$ & $\beta_{\text {tot }}$ & $\mu \beta$ & $\beta_{\mathrm{x}}$ & $\beta_{\mathrm{y}}$ & $\beta_{\mathrm{z}}$ & $\beta_{\text {tot }}$ & $\mu \beta$ \\
\hline D1 & 12706.87 & 186.45 & 232.229 & 12710.36 & $1101.10^{\mathrm{a}}$ & 13108.55 & 266.41 & 191.86 & 13112.66 & $1143.21^{\mathrm{a}}$ \\
\hline D2 & 7764.09 & 290.09 & 29.74 & 7769.57 & 809.59 & 7849.93 & 393.07 & 18.28 & 7859.79 & 819.99 \\
\hline D3 & -30725.84 & 753.14 & 238.27 & 30735.99 & 2774.25 & -33670.08 & 778.68 & 210.87 & 33679.74 & 3069.29 \\
\hline D4 & -16856.33 & -808.21 & -429.07 & 16881.15 & 1336.31 & -17501.07 & -832.48 & -379.11 & 17524.96 & 1447.20 \\
\hline D5 & -35544.96 & -1538.06 & 171.35 & 35578.63 & 3247.35 & -38538.16 & -1506.42 & 206.80 & 38568.15 & 3540.84 \\
\hline D6 & 25856.89 & -380.36 & -398.55 & 25862.76 & 2384.56 & 27771.85 & -419.35 & -340.01 & 27777.09 & 2572.23 \\
\hline D7 & -49212.86 & -2306.75 & 632.68 & 49270.95 & 4434.12 & -54032.18 & -2129.67 & 606.35 & 54077.54 & 5064.34 \\
\hline D8 & 41911.74 & 127.36 & -89.01 & 41912.03 & 5047.73 & 45412.67 & 191.59 & -90.55 & 45413.16 & 5510.60 \\
\hline
\end{tabular}

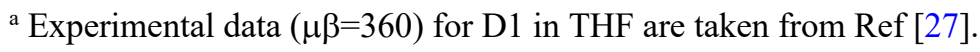


Table 5

\begin{tabular}{|c|c|c|c|c|c|c|c|c|c|c|c|}
\hline & Method & $\lambda_{\text {abs }}$ & $E_{\mathrm{ge}}$ & $f_{\mathrm{ge}}$ & Major MO contributions & $\mu_{\mathrm{ge}}{ }^{\mathrm{x}}$ & $\mu_{\mathrm{ge}}{ }^{\mathrm{y}}$ & $\mu_{\mathrm{ge}}^{\mathrm{z}}$ & $\mu_{\mathrm{ge}}$ & $\alpha_{\mathrm{CT}}$ & $\beta_{\mathrm{CT}}$ \\
\hline \multirow[t]{2}{*}{ D1 } & CAM-B3LYP & 401 & 3.091 & 0.592 & $\mathrm{H} \rightarrow \mathrm{L}(86 \%), \mathrm{H}-1 \rightarrow \mathrm{L}(8 \%)$ & 2.796 & -0.065 & 0.001 & 2.79 & 2.531 & 0.056 \\
\hline & M06-2X & 402 & 3.081 & 0.563 & $\mathrm{H} \rightarrow \mathrm{L}(90 \%), \mathrm{H}-1 \rightarrow \mathrm{L}(6 \%)$ & -2.729 & 0.055 & -0.003 & 2.73 & 2.419 & 0.053 \\
\hline \multirow[t]{2}{*}{ D2 } & CAM-B3LYP & 340 & 3.647 & 0.632 & $\mathrm{H} \rightarrow \mathrm{L}(71 \%), \mathrm{H}-1 \rightarrow \mathrm{L}(19 \%)$ & 2.514 & -0.863 & 0.031 & 2.65 & 1.938 & 0.035 \\
\hline & M06-2X & 338 & 3.663 & 0.601 & $\mathrm{H} \rightarrow \mathrm{L}(79 \%), \mathrm{H}-1 \rightarrow \mathrm{L}(10 \%)$ & 2.441 & -0.855 & 0.033 & 2.59 & 1.827 & 0.032 \\
\hline \multirow[t]{2}{*}{ D3 } & CAM-B3LYP ${ }^{b}$ & 405 & 3.059 & 1.498 & $\mathrm{H} \rightarrow \mathrm{L}(59 \%), \mathrm{H}-1 \rightarrow \mathrm{L}(18 \%)$ & -4.469 & 0.135 & -0.018 & 4.46 & 6.503 & 0.233 \\
\hline & M06-2X & 413 & 3.003 & 1.292 & $\mathrm{H} \rightarrow \mathrm{L}(72 \%), \mathrm{H} \rightarrow \mathrm{L}+1(9 \%)$ & 4.189 & -0.144 & 0.012 & 4.19 & 5.850 & 0.200 \\
\hline \multirow[t]{2}{*}{ D4 } & CAM-B3LYP & 421 & 2.948 & 0.708 & $\mathrm{H} \rightarrow \mathrm{L}(88 \%), \mathrm{H} \rightarrow \mathrm{L}+1(2 \%)$ & -3.115 & -0.321 & -0.003 & 3.13 & 3.327 & 0.087 \\
\hline & M06-2X & 422 & 2.939 & 0.688 & $\mathrm{H} \rightarrow \mathrm{L}(91 \%), \mathrm{H}-1 \rightarrow \mathrm{L}(4 \%)$ & -3.074 & -0.318 & -0.002 & 3.09 & 3.251 & 0.084 \\
\hline \multirow[t]{2}{*}{ D5 } & CAM-B3LYP & 436 & 2.846 & 1.564 & $\mathrm{H} \rightarrow \mathrm{L}(60 \%), \mathrm{H} \rightarrow \mathrm{L}+1(14 \%)$ & -4.728 & -0.269 & 0.064 & 4.74 & 7.883 & 0.321 \\
\hline & M06-2X & 442 & 2.807 & 1.387 & $\mathrm{H} \rightarrow \mathrm{L}(72 \%), \mathrm{H} \rightarrow \mathrm{L}+1(10 \%)$ & -4.484 & -0.235 & 0.052 & 4.49 & 7.181 & 0.281 \\
\hline \multirow[t]{2}{*}{ D6 } & CAM-B3LYP & 409 & 3.033 & 1.196 & $\mathrm{H} \rightarrow \mathrm{L}(65 \%), \mathrm{H} \rightarrow \mathrm{L}+1(10 \%)$ & 4.002 & -0.285 & -0.002 & 4.01 & 5.308 & 0.172 \\
\hline & M06-2X & 416 & 2.979 & 0.961 & $\mathrm{H} \rightarrow \mathrm{L}(78 \%), \mathrm{H} \rightarrow \mathrm{L}+1(8 \%)$ & 3.619 & -0.244 & 0.001 & 3.63 & 4.428 & 0.132 \\
\hline \multirow[t]{2}{*}{ D7 } & CAM-B3LYP & 448 & 2.765 & 1.786 & $\mathrm{H} \rightarrow \mathrm{L}(48 \%), \mathrm{H} \rightarrow \mathrm{L}+1(16 \%)$ & -5.121 & -0.371 & 0.070 & 5.14 & 9.539 & 0.434 \\
\hline & M06-2X & 457 & 2.712 & 1.487 & $\mathrm{H} \rightarrow \mathrm{L}(64 \%), \mathrm{H} \rightarrow \mathrm{L}+1(13 \%)$ & -4.722 & -0.285 & 0.053 & 4.73 & 8.251 & 0.353 \\
\hline \multirow[t]{2}{*}{ D8 } & CAM-B3LYP & 446 & 2.779 & 1.971 & $\mathrm{H} \rightarrow \mathrm{L}(29 \%), \mathrm{H} \rightarrow \mathrm{L}+1(23 \%)$ & 5.377 & -0.202 & -0.013 & 5.38 & 10.417 & 0.494 \\
\hline & M06-2X & 456 & 2.720 & 1.343 & $\mathrm{H} \rightarrow \mathrm{L}(53 \%), \mathrm{H} \rightarrow \mathrm{L}+1(21 \%)$ & -4.487 & 0.132 & 0.007 & 4.49 & 7.406 & 0.299 \\
\hline
\end{tabular}

${ }^{\mathrm{b}}$ Experimental data $\left(\lambda_{\mathrm{abs}}=443 \mathrm{~nm}\right)$ for D3 in THF are taken from Ref [28] 


\section{Table 6}

\begin{tabular}{lllllllc}
\hline & Method & $\lambda_{\text {flu }}$ & $\mathrm{E}_{\text {flu }}$ & $\mathrm{f}$ & $\mathrm{M}$ Mjor MO contributions & $\mathrm{SS}$ & $\mu_{\mathrm{E}}$ \\
\hline D1 & CAM-B3LYP & 532 & 2.333 & 0.843 & $\mathrm{H} \rightarrow \mathrm{L}(91 \%), \mathrm{H}-1 \rightarrow \mathrm{L}(5 \%)$ & 128 & 12.49 \\
& M06-2X & 536 & 2.314 & 0.815 & $\mathrm{H} \rightarrow \mathrm{L}(94 \%), \mathrm{H}-1 \rightarrow \mathrm{L}(3 \%)$ & 134 & 12.45 \\
D2 & CAM-B3LYP & 509 & 2.434 & 0.585 & $\mathrm{H} \rightarrow \mathrm{L}+1(85 \%), \mathrm{H}-1 \rightarrow \mathrm{L}(10 \%)$ & 169 & 15.01 \\
& M06-2X & 512 & 2.421 & 0.558 & $\mathrm{H} \rightarrow \mathrm{L}(89 \%), \mathrm{H}-1 \rightarrow \mathrm{L}(8 \%)$ & 172 & 14.92 \\
D3 & CAM-B3LYP & 545 & 2.275 & 1.879 & $\mathrm{H} \rightarrow \mathrm{L}(73 \%), \mathrm{H} \rightarrow \mathrm{L}+1(9 \%)$ & 140 & 12.48 \\
& M06-2X & 557 & 2.225 & 1.739 & $\mathrm{H} \rightarrow \mathrm{L}(82 \%), \mathrm{H} \rightarrow \mathrm{L}+1(6 \%)$ & 144 & 12.43 \\
D4 & CAM-B3LYP & 544 & 2.279 & 1.033 & $\mathrm{H} \rightarrow \mathrm{L}(91 \%), \mathrm{H}-1 \rightarrow \mathrm{L}(2 \%)$ & 123 & 11.69 \\
& M06-2X & 547 & 2.258 & 1.008 & $\mathrm{H} \rightarrow \mathrm{L}(94 \%), \mathrm{H}-1 \rightarrow \mathrm{L}(3 \%)$ & 125 & 11.63 \\
D5 & CAM-B3LYP & 581 & 2.133 & 2.039 & $\mathrm{H} \rightarrow \mathrm{L}(79 \%), \mathrm{H} \rightarrow \mathrm{L}+1(7 \%)$ & 145 & 13.25 \\
& M06-2X & 590 & 2.101 & 1.943 & $\mathrm{H} \rightarrow \mathrm{L}(86 \%), \mathrm{H} \rightarrow \mathrm{L}+1(5 \%)$ & 148 & 13.14 \\
D6 & CAM-B3LYP & 570 & 2.175 & 1.799 & $\mathrm{H} \rightarrow \mathrm{L}(77 \%), \mathrm{H} \rightarrow \mathrm{L}+1(7 \%)$ & 161 & 12.90 \\
& M06-2X & 582 & 2.131 & 1.651 & $\mathrm{H} \rightarrow \mathrm{L}(84 \%), \mathrm{H} \rightarrow \mathrm{L}+1(5 \%)$ & 166 & 12.75 \\
D7 & CAM-B3LYP & 636 & 1.951 & 2.291 & $\mathrm{H} \rightarrow \mathrm{L}(66 \%), \mathrm{H} \rightarrow \mathrm{L}+1(14 \%)$ & 188 & 13.31 \\
& M06-2X & 647 & 1.917 & 2.141 & $\mathrm{H} \rightarrow \mathrm{L}(76 \%), \mathrm{H} \rightarrow \mathrm{L}+1(10 \%)$ & 190 & 13.14 \\
D8 & CAM-B3LYP & 605 & 2.051 & 2.121 & $\mathrm{H} \rightarrow \mathrm{L}(43 \%), \mathrm{H} \rightarrow \mathrm{L}+1(27 \%)$ & 159 & 15.74 \\
& M06-2X & 618 & 2.007 & 1.849 & $\mathrm{H} \rightarrow \mathrm{L}(58 \%), \mathrm{H} \rightarrow \mathrm{L}+1(22 \%)$ & 162 & 15.76 \\
\hline
\end{tabular}

${ }^{\mathrm{c}}$ Experimental data $\left(\lambda_{\mathrm{flu}}=535 \mathrm{~nm}\right)$ for D3 in THF are taken from Ref [28] 
Table 7

\begin{tabular}{lllllll}
\hline & $\lambda_{\mathrm{h}}$ & $\lambda_{\mathrm{e}}$ & $\Delta \lambda$ & AIP & EA & $\eta$ \\
\hline D1 & 0.517 & 0.625 & 0.108 & 5.880 & 0.737 & 2.572 \\
D2 & 0.536 & 0.593 & 0.057 & 6.639 & 1.175 & 2.732 \\
D3 & 0.474 & 0.526 & 0.052 & 5.726 & 1.101 & 2.313 \\
D4 & 0.391 & 0.618 & 0.227 & 5.874 & 0.862 & 2.506 \\
D5 & 0.383 & 0.528 & 0.145 & 5.694 & 1.208 & 2.243 \\
D6 & 0.385 & 0.578 & 0.193 & 5.757 & 1.314 & 2.222 \\
D7 & 0.391 & 0.465 & 0.074 & 5.659 & 1.524 & 2.068 \\
D8 & 0.378 & 0.566 & 0.188 & 5.819 & 2.014 & 1.903 \\
Alq3 & 0.769 & 0.702 & 0.067 & 6.343 & 2.945 & 1.699 \\
TPD & 0.578 & 0.879 & - & 5.871 & 0.259 & 3.065 \\
\hline
\end{tabular}


Figures
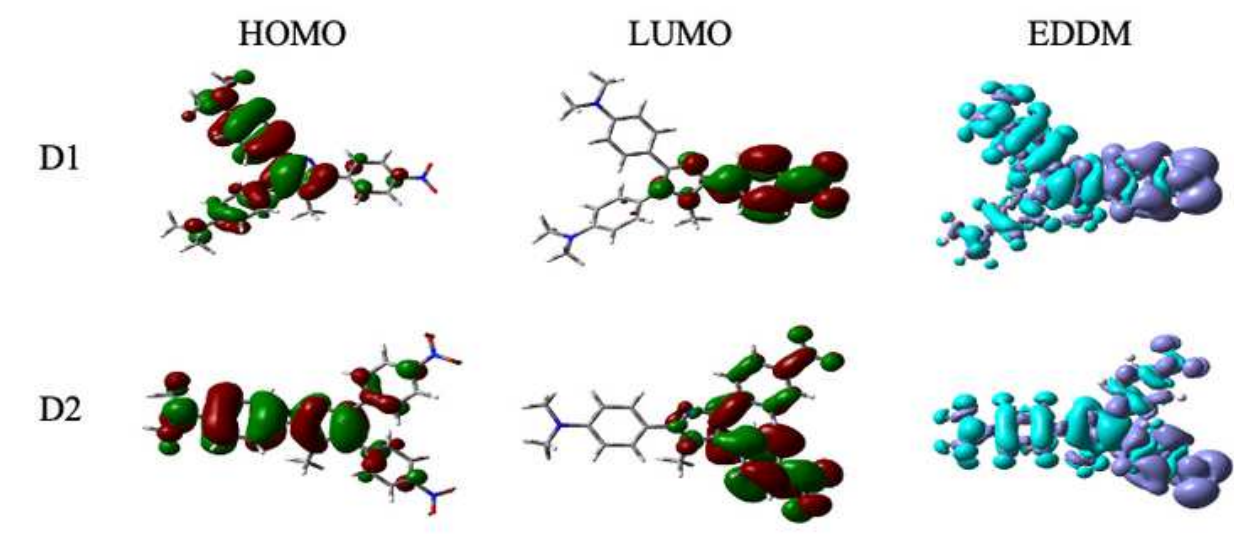

D3
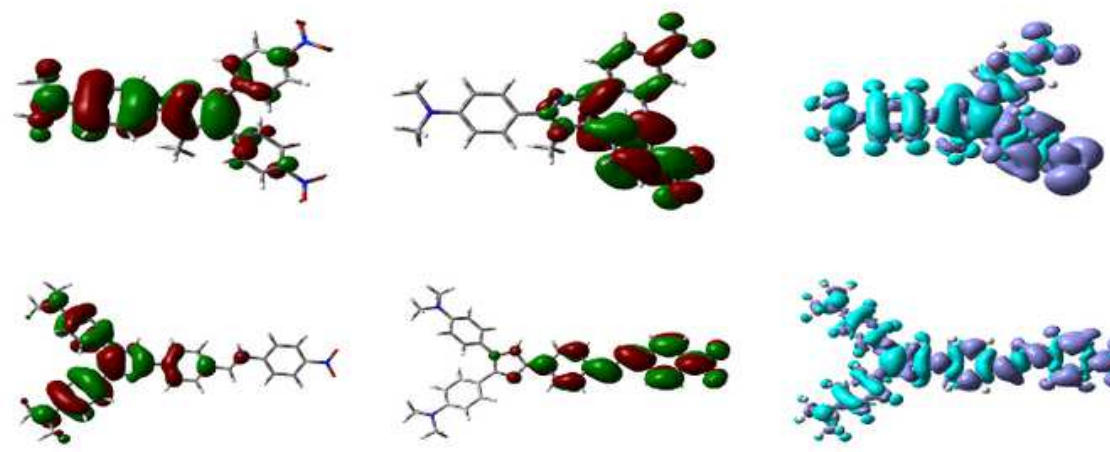

D4
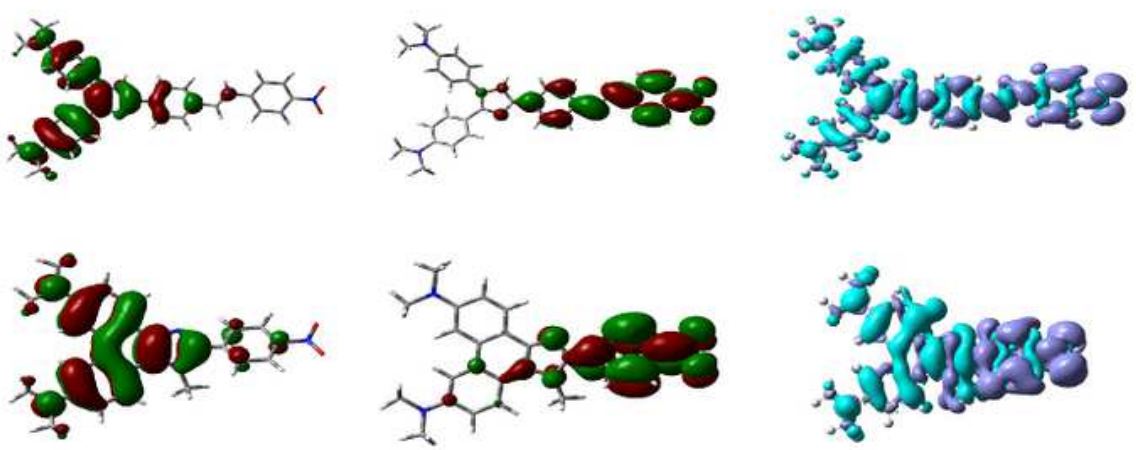

D5
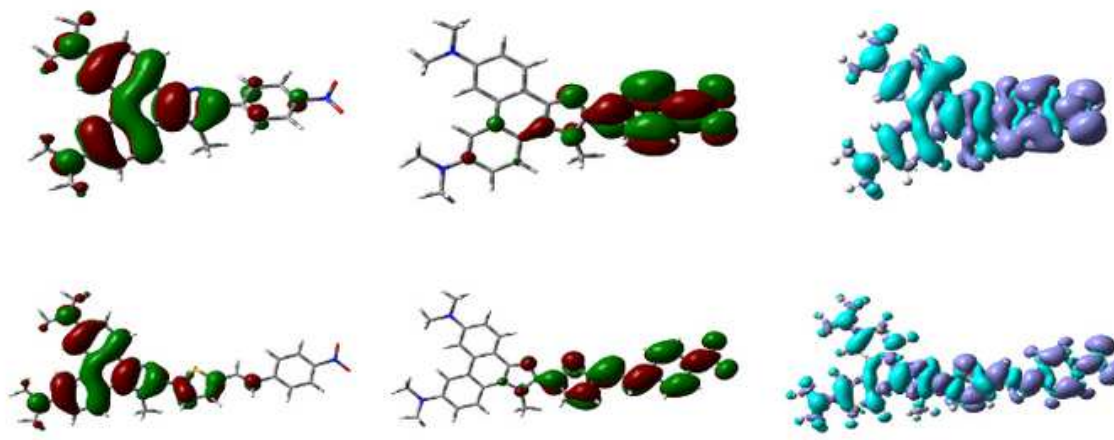

D6
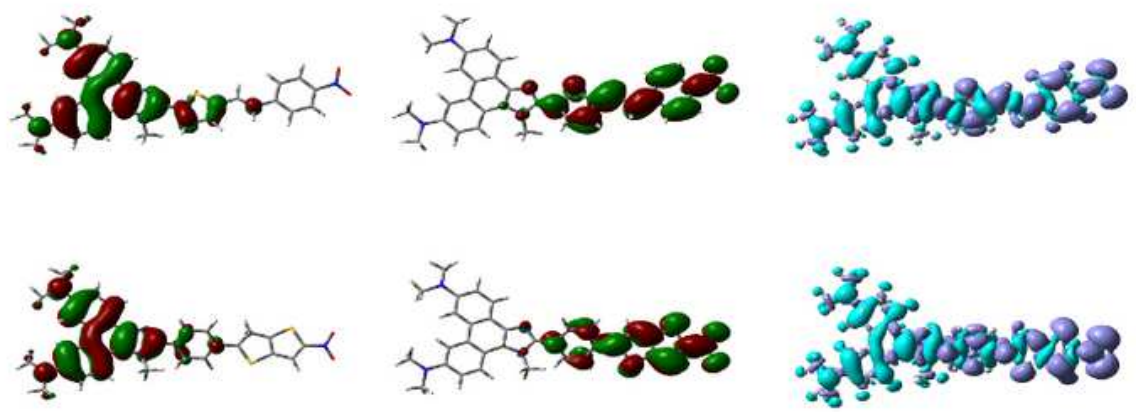

D7
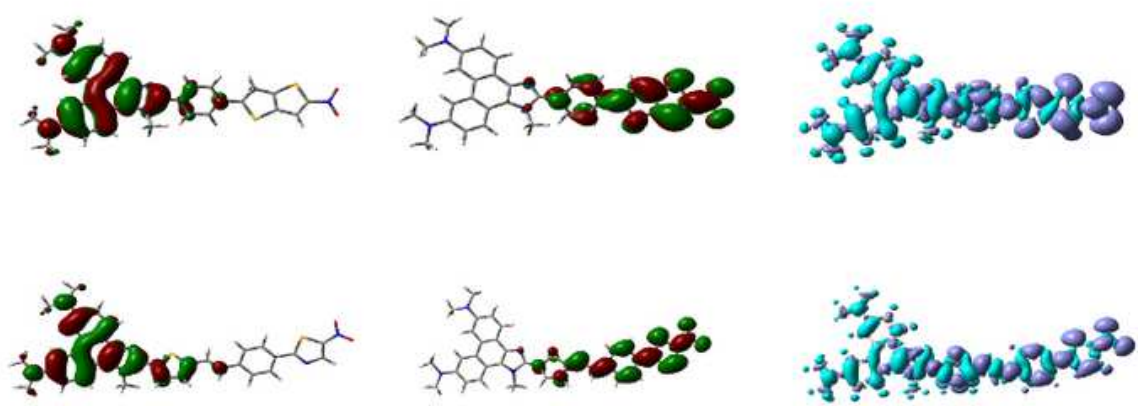

D8
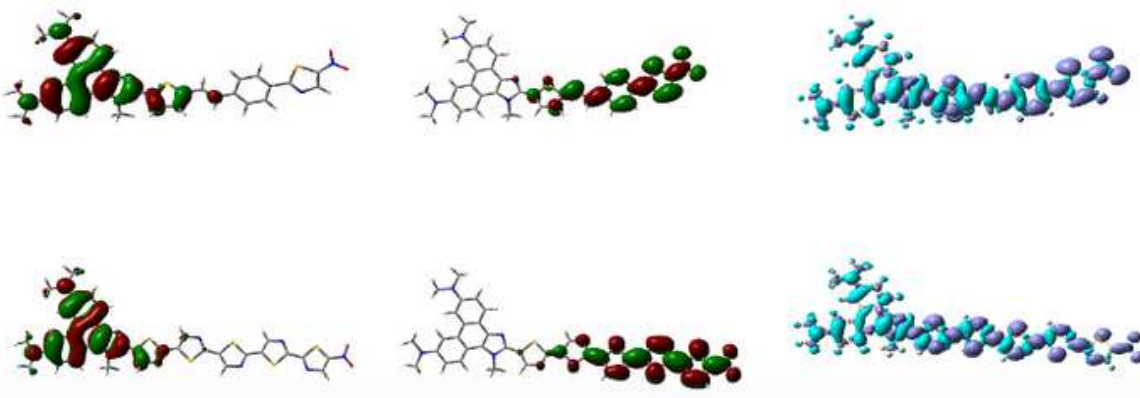

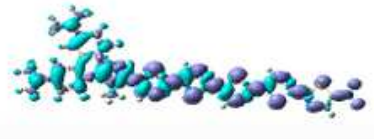

\section{Figure 1}

The frontier orbital plots of the HOMO and LUMO of chromophores D1-D8 in ground state. Electron density difference maps (EDDM) of compounds D1-D8. Cyan and purple colors indicate depletion and accumulation of electron density, respectively. 


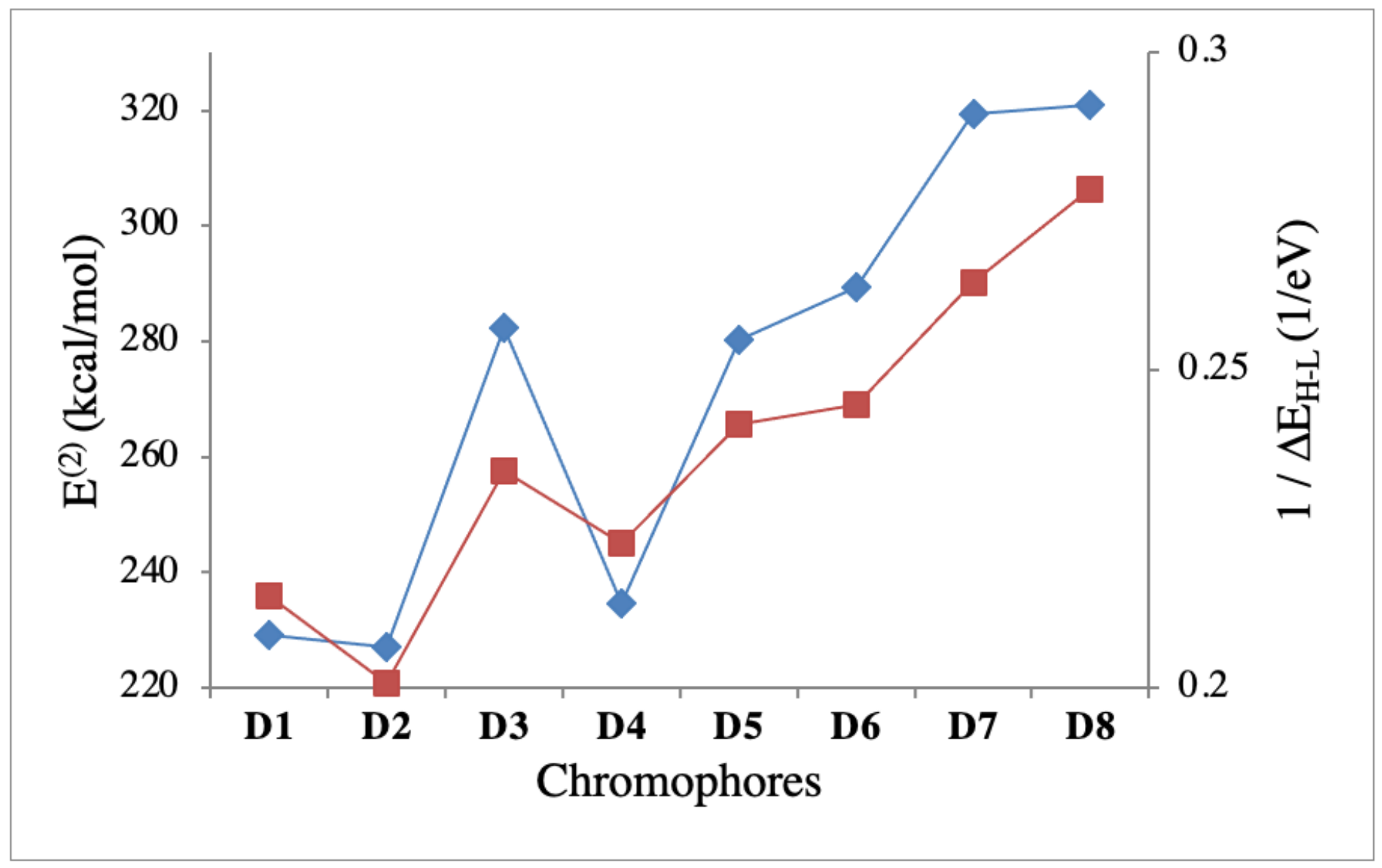

Figure 2

Correlation between $\mathrm{E}(2)$ (red line) and $\triangle \mathrm{EH}-\mathrm{L}$ (blue line) values for chromophores D1-D8. 

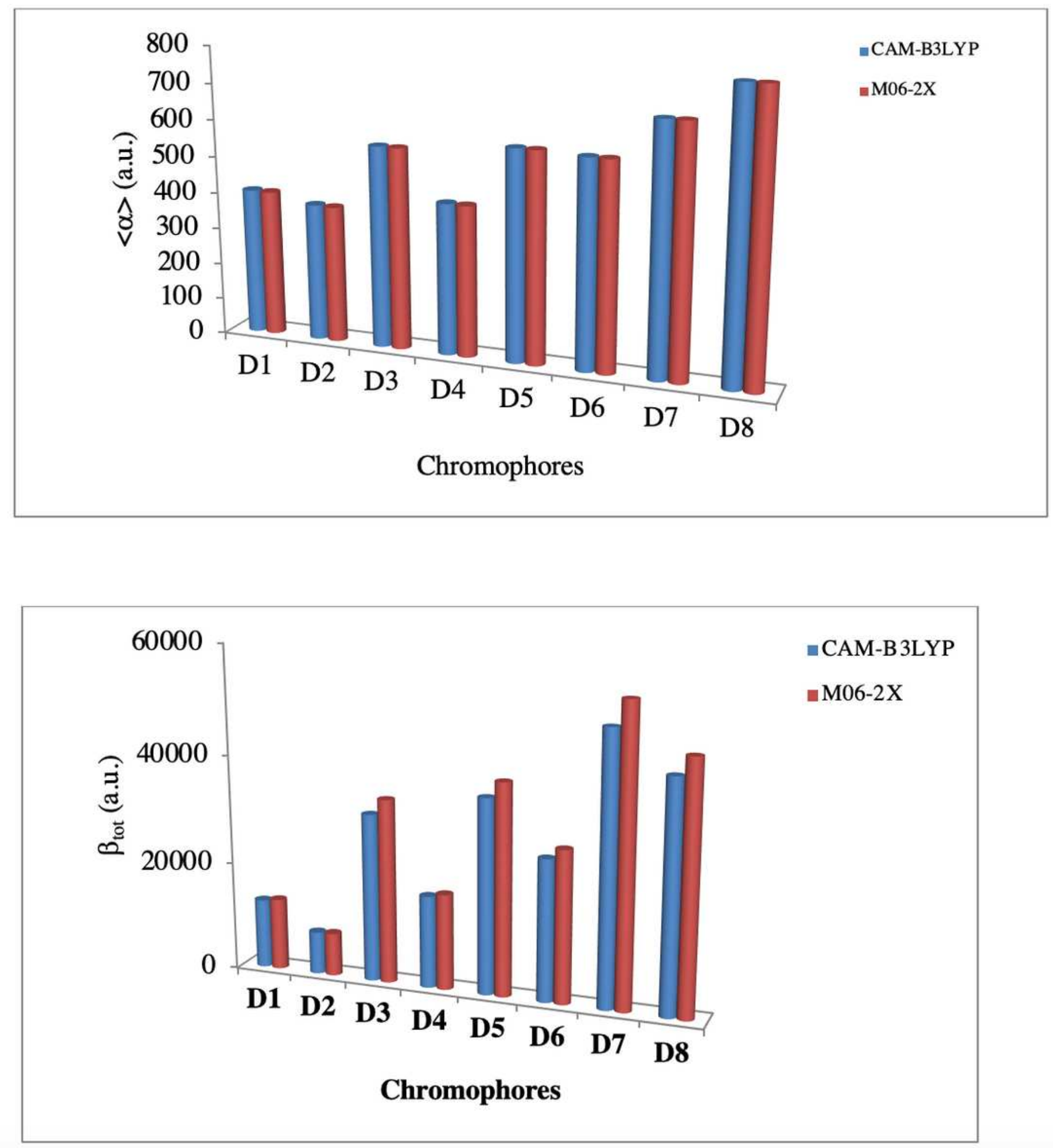

Figure 3

Comparison between CAM-B3LYP and M06-2X values of $<\mathrm{a}>$ and $\beta$ tot. 

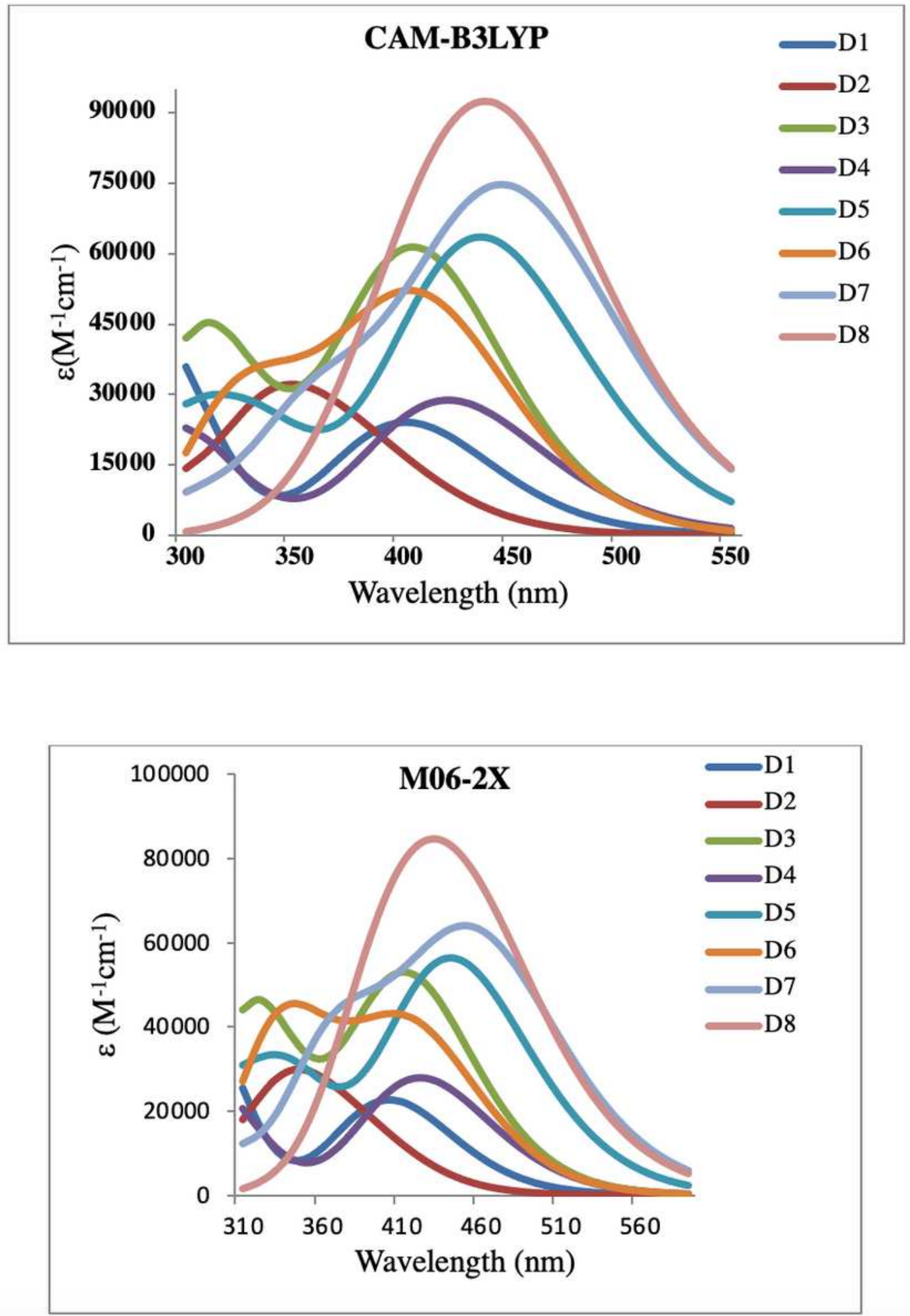

Figure 4

UV-Visible absorption spectra of chromophores D1-D8 in chloroform, calculated at TD-CAM-B3LYP/6$31+G(d, p)$ level and TD-M06-2X/6-31+G(d,p) levels. 

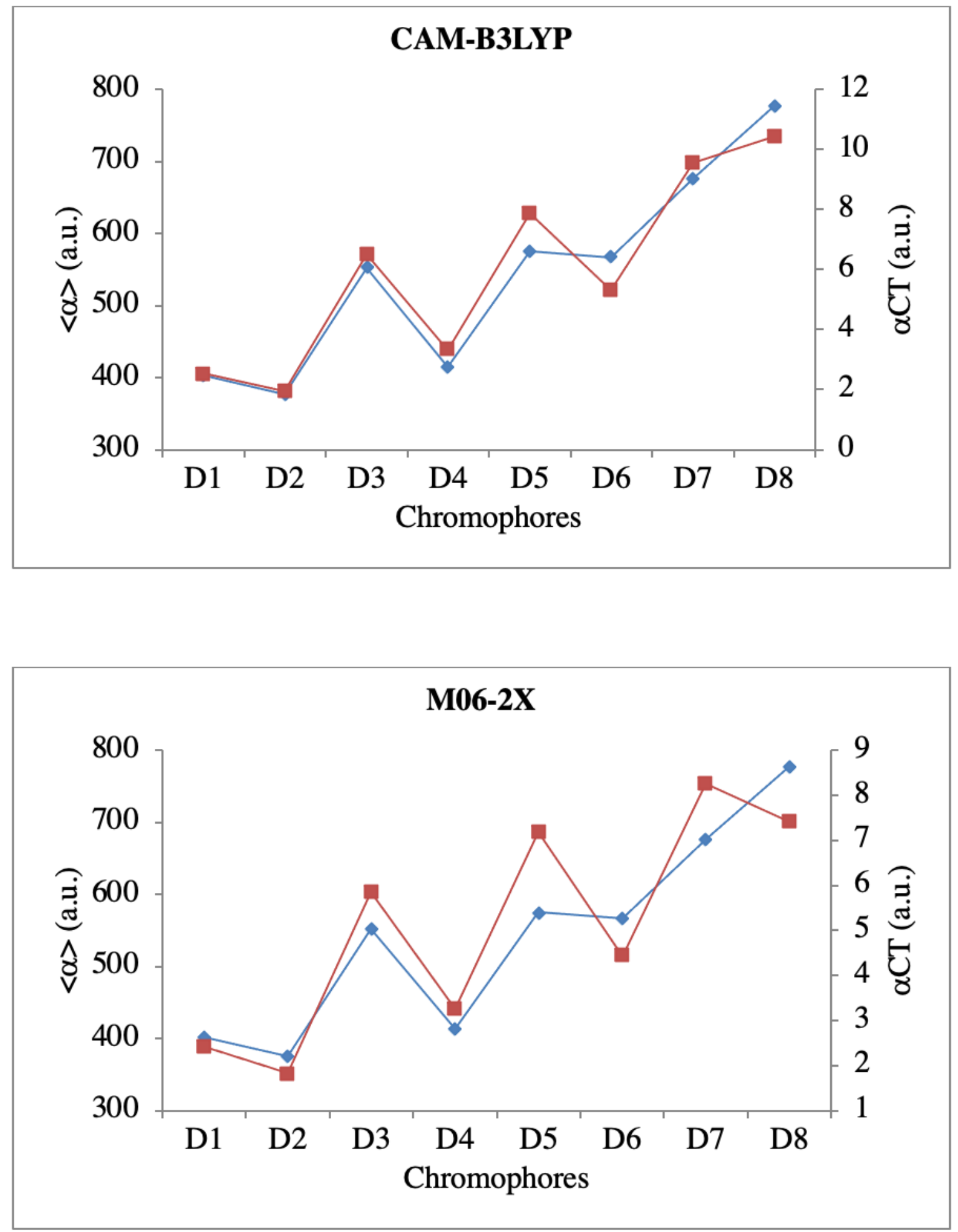

Figure 5

Correlation between $<a>$ values (blue line) and the corresponding aCT values (red line) for chromophores D1-D8. 

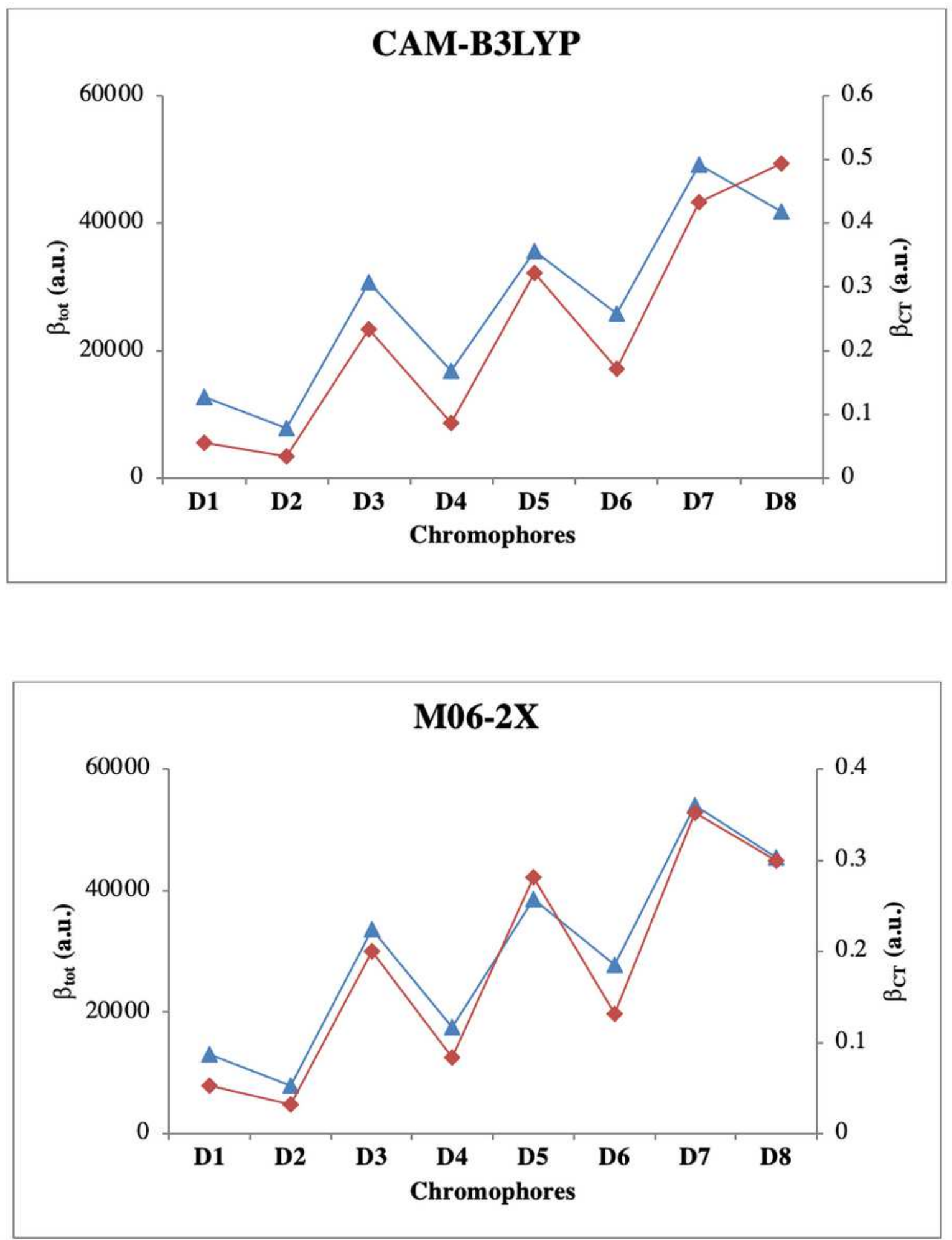

Figure 6

Correlation between the $\beta$ tot values (blue line) and the corresponding $\beta C T$ values (red line) for chromophores D1-D8. 

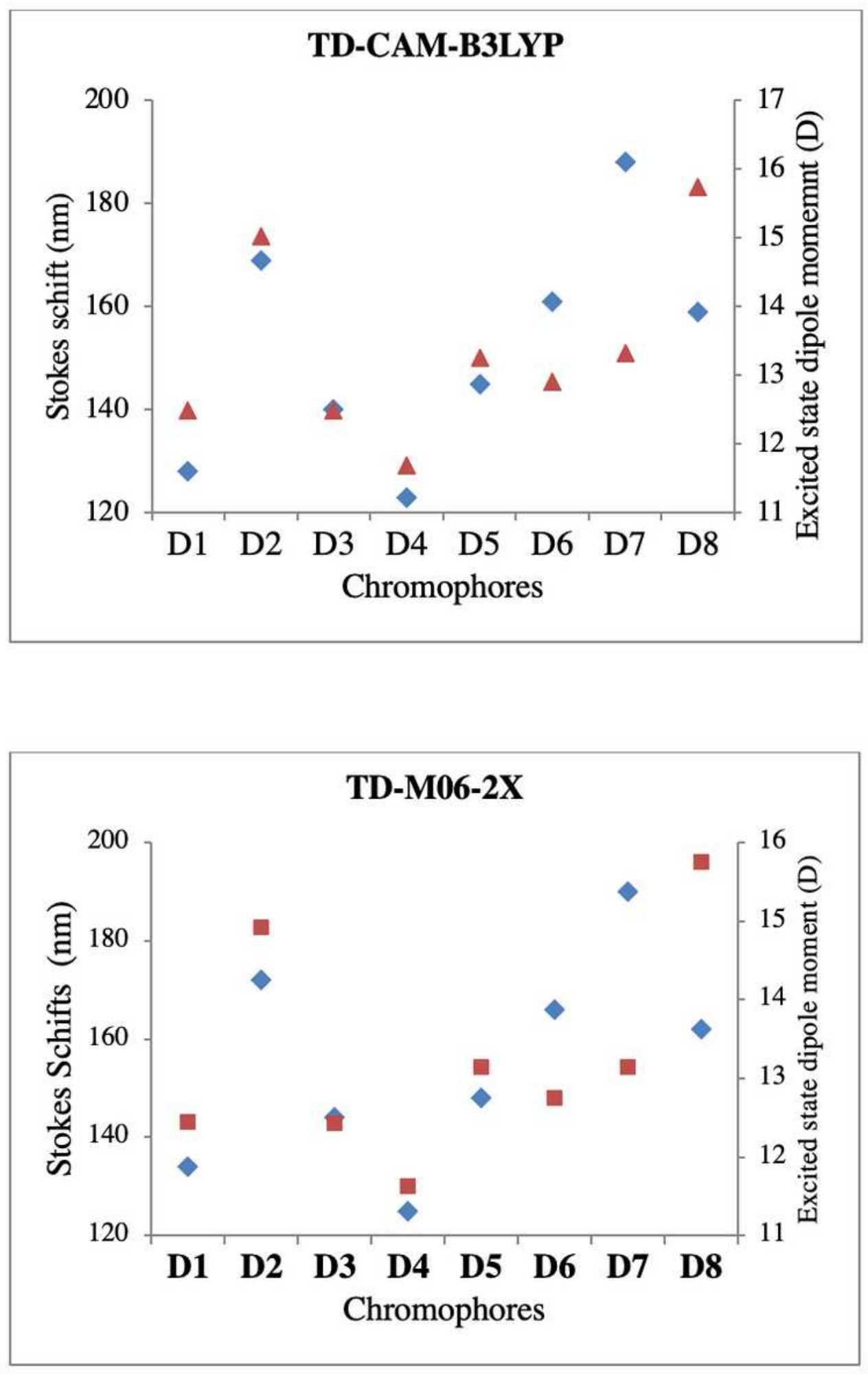

Figure 7

Correlation between Stokes shifts (nm) (blue) and excited state dipole moment $\mu \mathrm{E}(\mathrm{D})$ (red).

\section{Supplementary Files}

This is a list of supplementary files associated with this preprint. Click to download. 
- GraphicalAbstract.png

- Scheme1a.png

- Scheme1b.png

- attachmenttomanuscriptfinal.docx 